\title{
Transcriptional profiling of subcutaneous adipose tissue in Italian Large White pigs divergent for backfat thickness
}

\begin{tabular}{|r|l|}
\hline Journal: & Animal Genetics \\
\hline Manuscript ID & AnGen-15-06-0185.R2 \\
\hline Manuscript Type: & Full Paper \\
\hline Date Submitted by the Author: & n/a \\
\hline Complete List of Authors: & $\begin{array}{l}\text { Zambonelli, Paolo; Bologna University, Department of Agricultural and- } \\
\text { Food Sciences (DISTAL) } \\
\text { Gaffo, Enrico; University of Padova, Department of Molecular Medicine } \\
\text { Zappaterra, Martina; Bologna University, Department of Agricultural and- } \\
\text { Food Sciences (DISTAL) } \\
\text { Bortoluzzi, Stefania; University of Padova, Department of Molecular } \\
\text { Medicine } \\
\text { Davoli, Roberta; Bologna University, Department of Agricultural and-Food } \\
\text { Sciences (DISTAL) }\end{array}$ \\
\hline Keywords: & backfat, fat deposition, gene expression, differential analysis, pigs \\
\hline \multicolumn{2}{|c}{} \\
\hline
\end{tabular}

This is the preprint version of the accepted article, then published in Animal genetics, Volume 47, Issue 3, Pages 306-323 which has been published in final form at DOI https://doi.org/10.1111/age.12413

This article may be used for non-commercial purposes in accordance with Wiley Terms and Conditions for Use of Self-Archived Versions. 
1 Transcriptional profiling of subcutaneous adipose tissue in Italian

\section{Large White pigs divergent for backfat thickness}

\section{3}

4 Zambonelli Paolo ${ }^{1 *}$, Gaffo Enrico ${ }^{2 *}$, Zappaterra Martina ${ }^{1}$, Bortoluzzi Stefania ${ }^{2 \S}$, Davoli

5 Roberta $^{15}$

6

$7 \quad{ }^{1}$ Department of Agricultural and-Food Sciences (DISTAL), Bologna University, Via Fratelli

8 Rosselli 107, 42123 Reggio Emilia, Italy.

92 Department of Molecular Medicine, University of Padova, Via Gabelli 63, 35121 Padova,

10 Italy

11

$12{ }^{\S}$ To whom correspondence should be addressed. Roberta Davoli Tel. +39 051 2088050, Fax

13 +39051 2086172, roberta.davoli@unibo.it; Stefania Bortoluzzi Tel: +39 049 8276502, Fax:

14 +39049 8276209, stefania.bortoluzzi@unipd.it

15

16 *These authors contributed equally to this work 


\section{Summary}

19 Fat deposition is a widely studied trait in pigs for the implications with animal growth

20 efficiency, technological and nutritional characteristics of meat products, but the global

21 framework of the biological and molecular processes regulating fat deposition in pigs is still

22 incomplete. This paper describes the backfat tissue transcription profile in Italian Large

23 White pigs and reports genes differentially expressed between fat and lean animals

24 according to RNA-seq data. The backfat transcription profile was characterized by the

25 expression of 23,483 genes of which $54.1 \%$ were represented by known genes. Of 63,418

26 expressed transcripts, about $80 \%$ were non previously annotated isoforms. By comparing

27 the expression level of fat vs. lean pigs we detected 86 robust differentially expressed

28 transcripts, 72 more expressed (e.g. ACP5, BCL2A1, CCR1, CD163, CD1A, EGR2, ENPP1,

GPNMB, INHBB, LYZ, MSR1, OLR1, PIK3AP1, PLIN2, SPP1, SLC11A1, STC1) and 14 less

30 expressed (e.g. ADSSL1, CDO1, DNAJB1, HSPA1A, HSPA1B, HSPA2, HSPB8, IGFBP5, OLFML3)

31 in fat pigs. The main functional categories enriched in differentially expressed genes were

32 immune system process, response to stimulus, cell activation, and skeletal system

33 development, for the overexpressed, unfolded protein binding and stress response, for the

34 under-expressed genes, which include five heat shock proteins. Adipose tissue alterations

35 and impaired stress response are linked to inflammation and, in turn, to adipose tissue

36 secretory activity similarly to what is observed in human obesity. Our results open the

37 opportunity to identify biomarkers of carcass fat traits to improve pig production chain and

38 to identify genetic factors that regulate the observed differential expression.

40 Keywords: backfat, fat deposition, gene expression, differential analysis, pigs

$$
-2 \text { - }
$$




\section{Introduction}

43 Backfat deposition and fat traits are among the most important characters studied in pigs,

44 due to their strong relation with human nutrition of pig products and for the technological

45 characteristics of high quality Protected Designation of Origin (PDO) dry-cured hams. The

46 amount of fat laid on the external part of the pig body (subcutaneous fat or backfat) is of

47 extreme importance for growth performances, as the lesser is the deposed fat, the better

48 the growth performances. Regarding technological aspects related to the dry-cured high

49 quality products and meat industry, an adequate layer of fat is required for the seasoning

50 process of PDO products, like dry cured hams (Bosi \& Russo, 2004; Čandek-Potokar \& Škrlep,

$512012)$.

52 During the last decade, pig transcriptomic data have been obtained initially by expressed

53 sequence tag sequencing (Mikawa et al., 2004; Uenishi et al., 2004; Chen et al., 2006;

54 Gorodkin et al., 2007; Uenishi et al., 2007) and microarrays (Hornshøj et al., 2007; Ferraz et

55 al., 2008; Moon et al., 2009), which allowed the comparison of gene expression level in

56 several pig tissues. More recently, the RNA-seq approach was used to compare the

57 transcription profile of different pig fat tissues or different pig breeds (Chen et al., 2011; Li

58 et al., 2012; Corominas et al., 2013; Jiang et al., 2013; Zhou et al., 2013; Sodi et al., 2014;

59 Toedebush et al., 2014; Wang et al. 2014). The differentially expressed genes (DEG)

60 reported in these studies are useful to investigate the metabolic pathways activated by or

61 associated with an increased fat deposition in pig body. However, the large amount of data

62 produced and the results reported in literature are often hardly comparable because of

63 differences in the studied breeds; heterogeneous animals' ages; and fat deposition stages.

- 3 - 
64 Moreover, these researches identified several new genes and transcripts not reported in

65 swine or other species. To date, the global framework of the biological processes regulating

66 backfat deposition in pigs is still incomplete, and literature is poor of studies carried out on a

67 homogeneous sample of individuals of the same breed reared on the same environmental

68 conditions.

69 The objective of this research was to investigate the transcription profile of Italian Large

70 White (ILW) pig backfat tissue and to compare the transcriptome of animals reared in the

71 same herd and farming conditions and showing high (FAT) and low (LEAN) backfat thickness.

72 Moreover a first functional characterization of DEGs has been obtained to provide new

73 insights on genes, pathways and processes influencing the divergent aptitude of

74 subcutaneous adipose tissue deposition in ILW pigs.

76 Materials and methods

77 Samples collection and RNA extraction

78 We sampled twenty individuals from a purebred population of 949 ILW sib-tested pigs

79 provided by the Italian National Association of Pig Breeders (Associazione Nazionale

80 Allevatori Suini, ANAS, http://www.anas.it. Accessed 22 June 2015). All animals used in this

81 study were kept according to Italian and European law for pig production and all procedures

82 described were in compliance with national and European Union regulations for animal care

83 and slaughtering. The animals were reared on the ANAS Sib-Test genetic station from about

$8430 \mathrm{~kg}$ live weight to at least $155 \mathrm{~kg}$ live weight. For the genetic evaluation of a boar, full sib

85 triplets (two females and one castrated male) were farmed on the genetic station to be

86 performance tested. The formula and amount of the ration was the same for all. It was

$$
-4-
$$


87 based mainly on cereals and soybean, given in excess calculated using the "quasi ad libitum"

88 rule (a ration sufficiently abundant that $60 \%$ of pigs were able to ingest the full supplied

89 food). At the end of tests, animals were transported to a commercial abattoir located about

$9025 \mathrm{~km}$ far from the test station according to the Council Rule (EC) No 1/2005 on the

91 protection of animals during transport and related operations and amending Directives

92 64/432/EEC and 93/119/EC and Regulation (EC) No 1255/97. At slaughterhouse the pigs

93 were electrical stunned and bled in a lying position in agreement with the Council

94 Regulation (EC) No 1099/2009 on the protection of animals at the time of killing. All

95 slaughter procedures were monitored by the Veterinary team appointed by the Italian

96 Ministry of Health. Backfat samples were collected after slaughter, from 949 ILW pigs

97 slaughtered at an average hot carcass weight of $118.97 \mathrm{~kg}( \pm 0.29 \mathrm{SEM})$ and at an average

98 age of eight months during the years 2011 and 2012 in 27 different slaughtering days. The

99 collected samples were immediately frozen in liquid nitrogen and stored at $-80^{\circ} \mathrm{C}$ in a deep

100 freezer until RNA extraction. For the RNA-seq analysis we selected the animals according to

101 the estimated breeding value (EBV) for backfat thickness (BFT) calculated by ANAS as

102 described by Russo et al. (2000; 2008). EBVs were determined through a BLUP multiple-trait

103 animal model procedure (Henderson \& Quaas, 1976) using the BFT, measured in mm,

104 recorded post mortem in correspondence of the gluteus medius muscle. The model included

105 fixed effects of batch in test, sex, age at beginning of test, age of sow, weight at slaughter,

106 age at slaughter, and inbreeding coefficient as well as the random effects of litter, individual

107 permanent environment, and animal. Pigs' genetic merit for the BFT trait was calculated

108 taking into account the additive relationship matrix. EBVs were expressed as differences

109 from the genetic mean value for the considered trait in the year 1993. Backfat thickness

110 genetic index may present negative values because the value of the trait is referred to the $-5-$ 
111 fixed genetic base defined by ANAS as mean values of the pigs born in 1993 and considered

112 as "zero", so the more negative values indicate lower values of BFT. The animals were

113 selected to compose two groups of 10 pigs showing extreme and divergent characteristics

114 for the BFT EBV with respect to the larger population of the 949 pigs (Table 1). The twenty

115 animals considered for RNA-seq analysis were slaughtered in 12 dates, with 5 dates

116 common to both groups. The animals were selected also according to their pedigree, in

117 order to avoid the presence of full sibs in the considered groups. From now on the two

118 groups will be referred as FAT and LEAN samples.

119

120 RNA extraction, library preparation, sequencing

121 Total RNA was extracted with Trizol (Invitrogen) according to the manufacturer's

122 instruction. RNA extracted samples were quantified using a Nanodrop ND-1000

123 spectrophotometer (Nanodrop Technologies) and the quality of the RNA was assayed using

124 an Agilent 2100 BioAnalyzer (Agilent Technologies). The RNA libraries were prepared from

125 total RNA using the TruSeq RNA sample preparation kit (Illumina) and version 3 of the

126 reagents, following the manufacturer's suggested protocol. The libraries were tagged and

127 couples of libraries were run on a single lane of an Illumina HiSeq2000. Reads are $100 \mathrm{nt}$

128 paired-end represented in FASTQ format.

130 Architecture of the bioinformatics pipeline

131 A computational pipeline to process the sequencing data for gene/transcript expression

132 estimation and to perform differential expression analysis between the two sample groups

133 was developed. The pipeline components to achieve expression estimates were assembled

$$
-6-
$$


134 using Scons software (http://www.scons.org/. Accessed 22 June 2015), which allows the

135 parallelization and automation of the pipeline tasks. The pipeline and its following steps are

136 detailed in the next paragraphs.

137

138 RNA-seq data pre-processing and mapping to swine genome

139 Exploratory analyses on the raw reads quality were carried out using the FastQC v0.10.1

140 software (http://www.bioinformatics.babraham.ac.uk/projects/fastqc/. Accessed 22 June

141 2015), which generates an HTML report for each sample read set. Read fragments with

142 quality Phred score lower than 30 were trimmed using the DynamicTrim script of the

143 SolexaQA v2.1 (Cox et al., 2010). The FASTX-Toolkit v0.0.13.2

144 (http://hannonlab.cshl.edu/fastx_toolkit/. Accessed 22 June 2015) was used for trimming

145 result report. A custom Python script using the HTSeq package (Anders et al., 2015) filtered

146 out the trimmed reads shorter than 50 nucleotides. To maintain a consistent paired-end

147 read set, discarded read mates were also filtered out, despite their length and quality. Each

148 sample paired-end clean read set was mapped to the swine genome (Sscrofa10.2.70) by

149 Tophat v2.0.8 (Kim et al., 2013) using default parameters with transcriptome inference from

150 Ensembl annotation (Tophat2 used Bowtie v2.1.0.0; Langmead \& Salzberg, 2012) and

151 SAMtools v0.1.19()Li et al., 2009).

152

153 Gene/transcript expression evaluation and transcript reconstruction

154 Gene annotation for the reference genome was retrieved from Ensembl (BioMart) using the

155 biomaRt R package (Durinck et al., 2009). Read alignments were processed by Cufflinks

156 v2.1.1 (Roberts et al., 2011a; Roberts et al., 2011b; Trapnell et al., 2010) to identify and

$$
-7 \text { - }
$$

\section{Animal Genetics}


157 discover expressed genes and transcripts, and to quantify their expression. Expression data

158 were indicated as Fragments Per Kilobase of transcript per Million mapped reads (FPKM).

159 Cufflinks was applied to each sample alignment; then, we merged the transcript predictions

160 in a non-redundant reference using the Cuffmerge tool from the Cufflinks package. To

161 reduce artefacts deriving from the transcript prediction and normalisation strategies, only

162 predicted transcripts at least $200 \mathrm{nt}$ long and with minimal expression of 100 (Cufflinks

163 normalised) reads in at least one of the two groups were considered for transcriptome

164 reconstruction and for the following analyses.

165

166 Gene and transcript differential expression assessment

167 The samples were inspected by principal component analysis to examine their similarities.

168 The read counts of each gene in the 20 considered samples were transformed with the

169 variance stabilizing transformation function provided by the DESeq2 package (Anders \&

170 Huber, 2010) and used to compute the principal components.

171 The genes identified by Cufflinks were assessed for differential expression (DE) between the

172 LEAN and FAT groups, by means of two strategies, namely Cuffdiff2 (v2.1.1 from the

173 Cufflinks package; Trapnell et al., 2012) and DESeq2 v1.2.1 (Anders \& Huber, 2010). Instead,

174 transcript DE was assayed only with Cuffdiff2. To represent gene expression, the two

175 methods use similar statistical approaches based on generalized linear model (GLM) of the

176 negative binomial family. Cuffdiff 2 extends the model using a beta negative binomial

177 distribution to handle uncertainty of multi-mapped reads. On the contrary, DESeq2

178 considers only uniquely mapped reads (counted by means of the htseq-count script of the

179 HTSeq package (Anders et al., 2015), but facilitate the specification in the statistical model

$-8-$

Animal Genetics 
180 of additional factors effecting the fit of the GLM. In this study, the statistical model included

181 sex effect as a potential conditioning factor. Gene and transcript DE test computed P-values

182 were corrected according to the Benjamini-Hochberg procedure. Differentially expressed

183 genes and transcripts were considered statistically significant according to false discovery

184 rate less than or equal to 0.05 .

185

186 Transcript characterisation

187 Using custom scripts including BEDTools v2.17.0 software (Quinlan \& Hall, 2010), we

188 retrieved the nucleotide sequences of the transcripts extracting from the Sus scrofa genome

189 the stretches of nucleotides according to the annotation generated by the RNA-seq analysis

190 tools. Transcripts were identified or characterised by sequence similarity using BLASTN and

191 BLAST2 from the NCBI BLASTN suite

192 (http://blast.ncbi.nlm.nih.gov/Blast.cgi?PROGRAM=blastn\&PAGE_TYPE=BlastSearch\&LINK_L

193 OC=blasthome. Accessed 22 June 2015) using Megablast algorithm (Morgulis et al., 2008).

194 To assign a gene name, the sequences IDs obtained with this comparison were used to

195 query the NCBI Gene and the UniGene databases (http://www.ncbi.nlm.nih.gov/unigene/.

196 Accessed 22 June 2015). We used two strategies for transcript annotation. DE transcripts

197 and genes were annotated by similarity using $\mathrm{nr} / \mathrm{nt}$ nucleotide collection. The threshold

198 considered for the identification of our transcripts was identity $\geq 80 \%$ in at least $70 \%$ of the

199 sequence length of a transcript present in the database. Transcripts from new genes were

200 characterized using a comparative genomics approach. We compared the new transcripts

201 from intergenic regions with known human transcripts (RefSeq Release 72) by aligning with

202 BLASTN (NCBI BLAST 2.2.29+). For each transcript the best hit was considered, and then

$$
-9 \text { - }
$$

\section{Animal Genetics}


203 alignments with E-value greater than 10e-6, identity less than 60\%, and length less than 100

204 nucleotides were discarded.

205

206 Prediction of coding/non-coding potential

207 The transcript coding potential was predicted by CPC (Coding Potential Calculator; Kong et

208 al., 2007). CPC is a support vector machine-based classifier of transcript protein-coding

209 potential grounding on six features of sequence. Three features assess the extent and

210 quality of the predicted transcript ORF: the Framefinder software identifies the longest ORF

211 in the three forward and in the three reverse frames, then the coverage and the integrity of

212 the predicted ORF are evaluated. Another three features derive from results of BLASTX

213 search against UniProt Reference Clusters. All the features contribute together to a final

214 score, and to the classification of transcripts as coding or non-coding. Only transcripts not

215 including uncalled bases were considered for CPC analysis.

216

217 Validation by quantitative real time-PCR

218 The validation of selected RNA-seq results was performed using a quantitative real time-PCR

219 (qRT-PCR) approach using 18 out of the 20 samples used for the RNA-seq analysis. Two

220 samples, one in the FAT group and one in the LEAN group, were not considered because the

221 total RNA extracted was used completely for the RNA-seq analysis. QRT-PCR validation was

222 carried out using Rotor-Gene TM 6000 (Qiagen - Corbett Research). After DNase treatment

223 (TURBO DNA-free ${ }^{T M}$, Ambion, Applied Biosystems), $1 \mu \mathrm{g}$ of total RNA was reverse

224 transcribed using the iScript cDNA Synthesis kit (BIORAD) according to the manufacturers'

225 instructions.

-10 -

Animal Genetics 
226 The samples were first used to analyze four candidate normalizing genes beta-2-

227 microglobulin (B2M), polymerase (RNA) II (DNA directed) polypeptide A, 220kDa (POLR2A),

228 hypoxanthine phosphoribosyltransferase 1 (HPRT1), tyrosine 3-monooxygenase/tryptophan

229 5-monooxygenase activation protein, zeta (YWHAZ). The primer pairs and the PCR

230 conditions used are reported in Supplementary Table 1. The expression levels of these four

231 genes were evaluated using NormFinder and B2M and HPRT1, the two most stably

232 expressed normalizing genes, were utilized as reference genes. For each gene selected for

233 validation, we designed an external primer pair to obtain the amplicon for the standard

234 curve construction and an internal primer pair for the qRT-PCR on Rotor Gene 6000 (Table

235 S1). Standard curves for each gene were generated from $10-12$ serial dilutions (from $10^{9}$ to

23625 molecules/ $\mu$ l) of the PCR amplicons obtained with the external primer pairs and

237 containing the internal primers used in the qRT-PCR analysis. Amplifications were performed

238 in a total volume of $10 \mu$ l containing using $5 \mu$ l of the SYBR ${ }^{\circledR}$ Premix Ex Taq $^{\text {TM }}$ (Takara Bio

239 Inc.), $0.5 \mu$ l of each primer and about $100 \mathrm{ng}$ of cDNA. The used Premix Ex Taq ${ }^{\mathrm{TM}}$ is optimized

240 for a two-step cycling, and the amplification conditions for the tested genes are reported in

241 Table S1. The PCR efficiency was calculated as E=10 exp(-1/slope), with a range between -

2422.7 and -4.3 , indicating a good PCR efficiency result. All the PCR products were checked on a

243 polyacrylamide gel and the specificity of the amplification was checked by a final melting

244 curve analysis.

245 Threshold cycles obtained for the samples were converted by Rotor Gene 6000 to mRNA

246 molecules/ $\mu$ l using for each gene the relative standard curve (Bustin \& Nolan, 2004).

247 Moreover, the average mRNA molecules/ $\mu$ for each sample was normalized dividing the

248 mRNA molecules of a gene / $\mu$ l by the geometric average of B2M and HPRT1 mRNA

249 molecules/ $\mu$ in the given sample, as suggested by Bustin \& Nolan, 2004 and Vandesompele

$$
-11-
$$


250 et al., 2002. Differences on the expression level calculated for FAT and LEAN samples were

251 tested by two-tailed Student's t test. Statistical analyses were performed with SAS version

2529.3 (SAS 9.3 Help and Documentation, Cary, NC. SAS Institute Inc.) and nominal P-value

$253 \leq 0.05$ was considered as significance threshold.

254

255 Functional characterization

256 Functional annotation, classification and annotation clustering of selected gene sets were

257 carried out by DAVID Tools 6.7 (Huang et al., 2009) using Biological Processes, Molecular

258 Function gene ontology categories and KEGG pathways. A threshold for significance of

$259 \mathrm{P}<0.01$ and $\mathrm{P}<0.05$ after Benjamini correction was considered for the selection of the

260 functional categories respectively in the characterization of most expressed transcripts and

261 for the selection of the functional categories of DEG.

262

263 Results

264 Samples

265 In this study we applied RNA-seq by Illumina technology to the study of gene expression in

266 backfat tissue of 20 ILW pigs. We considered a large group of 949 sampled animals, with

267 EBV for BFT ranging from $-10.64 \mathrm{~mm}$ to $7.28 \mathrm{~mm}$, with mean value and standard deviation

268 (SD) $-1.96 \mathrm{~mm}$ and 3.01, respectively. We selected, from the whole collected population,

269 two groups of 10 unrelated pigs (FAT and LEAN) with extremely divergent EBVs for BFT, with

270 1:1 sex ratio within each group. The mean values of each of the two selected groups of pigs

271 are outside the range $-7.98 \mathrm{~mm} / 4.06 \mathrm{~mm}$ defined by the mean value of the 949 samples \pm 2

-12 -

Animal Genetics 
272 standard deviations. Specifically, FAT and LEAN animals were associated to average BFT

273 values of $+5.22 \mathrm{~mm}( \pm 1.30 \mathrm{SD})$ and $-8.63 \mathrm{~mm}( \pm 1.40 \mathrm{SD})$ as indicated in Table 1.

274

275 Sequencing, reads pre-processing and mapping

276 Pairs of samples were run together, after barcoding, on a single lane of an Illumina HiSeq

2772000 apparatus, obtaining a total of 3,917,123,414 raw reads for the 20 considered samples,

278 with an average of 195,856,171 raw reads per sample (Table S2; GEO accession GSE68007).

279 After trimming and length filtering the clean reads per sample were on the average

$280113,934,264$ (58.04\%) and were used for read-to-genome mapping (Figure S1A). Reads that

281 align on a single genome locus (uniquely mapped reads) were on the average the $91.07 \%$ of

282 the mapped reads (Table S2). The $72.42 \%$ of the uniquely mapped reads $(72,219,306.45$ on

283 the average aligned to annotated exons, the $19.15 \%$ mapped on intergenic regions and the

$2848.43 \%$ mapped on introns of annotated genes. The deep sequencing allowed the

285 identification of genes expressed at low level and relatively rare alternatively spliced

286 transcripts. We observed splicing events in the $21.19 \%$ of the reads on the average,

287 providing useful information for the reconstruction of alternative transcript isoforms (Figure 288 S1B).

289

290 Transcripts and genes expressed in backfat samples

291 The deep sequencing analysis of backfat transcripts performed on two groups of pigs

292 divergent for fat deposition in this tissue allowed the detection of 63,418 transcripts. Many

293 of them have not yet been annotated in the porcine genome, thus providing new consistent

294 resources for pig genome annotation and studies of adipose tissue biology. We identified

$$
-13-
$$


295 the expression of genes on all porcine autosomes, sex chromosomes and mitochondrial

296 genome. Chromosome 1 has the largest number of expressed genes (8.23\%), followed by

297 chromosomes 6 (7.84\%) and 2 (7.25\%). Furthermore, a non-negligible part (12.48\%) of the

298 expressed genes is located in genomic scaffolds (Figure S1C), as about the $7.5 \%$ of the

299 genome has no assigned location yet, as described in Ensembl annotation of pig genome

300 (database version 78 at the time of the analysis ;

301 http://www.ensembl.org/Sus_scrofa/Location/Genome. Accessed 22 June 2015). In term of

302 genes, we identified 23,483 expressed pig genes: 12,707 known and 10,776 putative new

303 genes.

304 Transcripts were split in different classes according to their matching with the genome

305 annotations (Figure 1A, Table S3). Transcripts matching exactly the reference annotation are

306 indicated as "known" transcripts; annotated transcripts' new isoforms or overlapping with

307 annotated transcript are indicated as "novel isoforms; and all other transcripts, such as

308 those expressed from extragenic regions, are referred as "new" transcripts and might

309 represent putative new genes. The majority of expressed transcripts are novel isoforms

310 (35,030; the $55.2 \%)$ or known transcripts $(12,969$, representing the $20.5 \%)$ that are

311 prevalently annotated as protein coding $(12,883 ; 99.3 \%)$. The expressed new transcripts are

$31215,419(24.3 \%)$.

313 Transcript lengths range from 200 to $50,610 \mathrm{nt}$, with median and average values of 3,224

314 and 3,979. Average size exceeds the $2 \mathrm{~kb}$ pig mean transcript size that can be estimated

315 according to Ensembl pig coding transcript annotation. We observed that the novel isoforms

316 reconstructed are longer than "known" pig transcripts (Figure 1B).

$-14-$ 
317 Sequences longer than $5 \mathrm{~kb}$ compose the $25 \%$ of the expressed transcripts. Noteworthy, we

318 detected two transcripts overlapping ZBTB16 gene and two new transcripts from

319 chromosome 16 that are longer than $40 \mathrm{~kb}$.

320 Considering transcripts expression, we observed that new transcripts are less expressed in

321 fat tissue than known transcripts (Figure 1C). Nevertheless, all the three transcript

322 categories span a considerably large range of expression values.

323 The majority of the expressed genes $(12,138 ; 52 \%)$ present only one transcript isoform

324 expressed in fat tissue (Figure 1D); the $27.0 \%$ and the $18.3 \%$ of the genes present two and

325 three expressed isoforms, respectively, whereas the remaining $12.7 \%$ of the genes are

326 associated each one to 4 to 31 different isoforms. We identified 31 isoforms for the gene

327 MAP4K4, for which a complex expression pattern is reported in humans: Ensembl release 79

328 lists 20 MAP4K4 transcripts generated by at least 3 different promoters, by complex

329 alternative splicing and by polyadenylation patterns, whereas five protein isoforms are

330 reported in UniProt release 2015_3.

331 Looking at isoform types, Figure 1E shows that many genes expressing only one transcript

332 (first bar from the left) in fat tissue are putative new genes (green portion). Interestingly,

333 some genes expressing only one transcript in fat tissue are represented only by a novel

334 isoform (first bar, red shadows). The proportion of novel isoforms (red portion) increases

335 along with the numbers of expressed transcripts per gene. Moreover, the transcripts classes

336 showing exonic overlap compared to a reference transcript are found in genes with a

337 varying number of transcripts and are particularly abundant in genes with up to three

338 isoforms. The remaining transcript classes are very rare.

339 Interesting new isoforms derived from known genes regard Perilipin 2 (PLIN2; alias ADFP,

340 adipofilin), an important gene for fat metabolism in pigs (Davoli et al., 2010; Gandolfi et al.,

$$
-15-
$$


341 2011) whose expression in humans correlates positively with cytosolic triacylglicerol levels

342 (Conte et al., 2013). Only one transcript is currently annotated in Ensembl for pig PLIN2

343 (ENSSSCT00000005701), whereas according to our results, PLIN2 expressed four different

344 isoforms. The most expressed PLIN2 transcript (expressed two times more in FAT than in

345 LEAN pigs) is a non-annotated isoform (TCONS_00002441 in Table 2; 2441DE in Figure S3)

346 characterized by the skipping of the fourth exon. The same transcript has also a shorter 3'

347 sequence with respect to the canonical PLIN2/ADFP form, probably due to the use of an

348 alternative polyadenylation site. Importantly, the skipping of the $83 \mathrm{nt}$ long exon four

349 introduces downstream a shift in the reading frame and a premature stop codon. Thus, this

350 transcript encodes a truncated protein (only 80 aa) corresponding to the $\mathrm{N}$-terminal region

351 and of the Perilipin domain of the PLIN2 protein annotated isoform (463 aa). The other two

352 new transcripts differ from the annotated isoform, one for the skipping of exon 2, and the

353 other for a longer first exon, probably due to alternative TSS usage by different promoters.

354 The four expressed isoforms are also heterogeneous in the length of the 3' UTR region.

355

356 Coding and non-coding transcripts from new genes

357 We obtained a characterization of intergenic transcripts from new genes first both by

358 similarity, comparing them against human transcripts, and by predicting their coding

359 potential. New pig transcripts with an assigned human best hit were 10,020 (65\%),

360 expressed by 7,099 genes (66\%), and corresponding to 4633 human Refseq sequences

$361(3,882$ unique gene symbols; Table S4).

362 We considered 12,702 intergenic transcripts for protein coding potential analysis. For each

363 transcript, the coding potential of both the forward and the reverse complement sequence

$-16-$

Animal Genetics 
364 were evaluated. According to CPC results, we classified the $35.8 \%(4,551)$ of transcripts as

365 coding, and the $64.2 \%(8,151)$ as non-coding. As done by Zhou et al., $(2014)$, we considered

366 as proper non-coding only those transcripts classified as non-coding and having a CPC score

367 lower than -1 for both the forward and the reverse sequence. A portion of the non-coding

368 transcripts (37.5\%) resulted with CPC score $<-1$ for both the forward and the reverse

369 complement sequences. We refer to these transcripts as "reliable non-coding" class, which

370 represented $24 \%(3,056)$ of the intergenic transcripts (Figure $2 \mathrm{~A})$. We observed that

371 intergenic coding transcripts are on average longer than intergenic non-coding transcripts

$372(4,149$ and 3,083 $\mathrm{nt}$, respectively), and that the reliable non-coding fraction has a even

373 shorter average length (2,571 nt; Figure 2B and Table S5). Reportedly, non-coding

374 transcripts tend to be shorter and to have fewer exons than coding transcripts in

375 mammalian genomes (lyer et al., 2015).

376 Coding transcripts have an average expression in fat tissue higher than the non-coding

377 transcripts (5.32 and 2.28 FPKM respectively, and 3.23 FPKM for the reliable non-coding

378 group; Figure 2C). One reliable non-coding transcript is ranked within the 100 most

379 expressed transcripts detected in backfat tissue; 15 reliable non-coding transcripts are

380 within the 1,000 most expressed transcripts; and 98 are within the $10 \%$ most expressed

381 transcripts (Table S6). In agreement with previous results showing that coding transcripts

382 tend to present higher expression than non-coding ones (Cabili et al., 2011; lyer et al.,

383 2015), we observe that intergenic transcripts ranking in the $10 \%$ most expressed in backfat

384 tissue are enriched in the coding category (55\%) and particularly if compared with the

385 proportion of the coding category within the set of intergenic transcripts (35.8\%; Figure 2D,

386 green portions).

$-17-$ 
388 Function of most expressed transcripts

389 A global view of the transcription profile of porcine backfat tissue was obtained by

390 averaging the FPKM values of all 20 analysed samples. The 1411 most expressed transcripts,

391 accounting together for $75 \%$ of expression, were chosen to extract the most expressed

392 genes (Table S6).

393 Among these genes, 59 are indicated as reliable non-coding (CPC score $<1$ ) and 66 showing a

394 positive CPC score are indicated as putative coding.

395 According to DAVID functional annotation and clustering, we characterized the biological

396 processes (Table S7) associated to the most expressed genes. Ribosomal activity, oxidative

397 phosphorylation, protein metabolic processes, intracellular protein transport, regulation of

398 translation initiation, fatty acid metabolism, response to oxidative stress resulted to be the

399 biological processes more represented in subcutaneous adipose tissue of the analysed

400 samples.

401

402 Gene/transcript differential expression

403 Unsupervised analysis of gene expression profiles was carried out to inspect similarities

404 among the samples. Principal component analysis revealed a clear separation of the LEAN

405 and FAT samples according to the first two most informative components (Figure S4 A),

406 which, notably, do not separate the samples by sex (Figure S4 B).

407 Average gene expression values for FAT and LEAN groups were 32.46 and 33.63 FPKM). In

408 both groups, few highly expressed genes contribute to the majority of the cumulative

409 expression. For instance, roughly $25 \%$ of expressed genes $(5,908$ and 5,728 in FAT and LEAN,

410 respectively) constitute $95 \%$ of the total detected expression (Figure S2). As expected,

-18 -

Animal Genetics 
411 transcript expression distribution is similar to the gene expression distribution being

412 positively skewed, with mean and median corresponding to 11.84 and 0.64 FPKM,

413 respectively. Transcripts average expression values are lower than genes expression values

414 since the latter was computed as the sum of transcripts expression of each gene.

415 To identify a set of robust DEG and DET the transcription profiles of FAT and LEAN samples

416 were compared with the integration of two methods applied at gene and at transcript

417 levels. Cuffdiff2 identified 414 DEGs between FAT and LEAN groups, corresponding to 1,187

418 transcripts: 266 DEGs are more expressed and 148 DEGs are less expressed in FAT samples.

419 Fold changes in base two logarithmic scale of DEGs range from 0.46 to 8.95 for the higher

420 expressed genes, and from -6.19 to -0.47 for the less expressed ones (Table S8). DESeq2

421 identified 586 DEGs (185 in common with the DEGs identified by Cuffdiff2) corresponding to

4221,504 transcripts: 358 genes are up-regulated and 228 genes are less expressed in FAT

423 samples. DEGs base two logarithmic scale transformed fold changes ( $\left.\log _{2} \mathrm{FC}\right)$ range from -

4241.13 to -0.20 for the less expressed genes and from 0.21 to 1.18 for the higher expressed

425 genes (Table S9). Cuffdiff2 differential expression analysis at the transcript-level identified

$426154 \mathrm{DE}$ transcripts (corresponding to 153 genes): 48 were less expressed and 106 transcripts

427 were more expressed in FAT samples, with $\log _{2}$ FC ranging from -3.44 to -0.54 and from

4280.64 to 3.66 , respectively (Table S10). On the whole, 818 genes were DE, or associated to at

429 least one DE transcript, according to at least one method, were detected (Figure 3A).

430 The overlapping of the different lists of DEGs and the list of DE transcripts (DET) evidenced a

431 group of 86 DET that are identified by all the approaches, from now on referred as

432 "common DET" (cDET). These DET belongs to 78 DEG, from now on referred as "common

433 DEG" (cDEG) since five genes are represented by more than one isoform (Table 2).

-19 - 
434 The cDET present the same fold change sign of the corresponding CDEG (Figure 3B): 72 DET

435 were more expressed in FAT (max Cuffdiff2 gene-level Log $_{2}$ FC 2.55 for DSC2 gene) and 14

436 DET were less expressed in FAT (minimum $\log _{2}$ FC -3.44 for an intergenic gene located in

437 GL894890.1 scaffold). Among the 86 cDET, 44 are known transcripts, 16 are novel isoforms

438 and 26 come from intergenic regions.

439 cDEG are found in all chromosomes except for chromosomes 16 and $\mathrm{Y}$, with up to $11 \mathrm{DE}$

440 genes in chromosome 4 and 19 DE genes in scaffolds (Figure 3C). The most expressed

441 (average FPKM greater than 100) known cDEG, reported in decreasing FPKM order, are

442 DNAJB1, CTSH, CTGF, C1QC, SPP1 and CDO1.

443

444 Coding and con-coding intergenic DET

445 We considered the 41 novel isoforms or new transcript CDET for CPC analysis. In 14 of these

446 transcripts both the forward and reverse sequence is probably non-coding, according to

447 integrated ORF analyses and to similarity searches, and to CPC score thresholds used before.

448 Five CDET with CPC score <-1 were scored as "reliable non-coding". Of the remaining

449 transcripts, nine presented low coding potential both in the forward and in the reverse

450 complement sequence but with CPC score ranging from -1 to 0 ("non-coding"), and 27 were

451 classified as coding transcripts (Table S11).

452

453 qRT-PCR confirmation of DE for selected genes

454 To validate the results obtained by RNA-seq, eleven cDEG were chosen according to the

455 absolute value of the $\log _{2}$ FC between FAT and LEAN pigs, or for their functional role and

456 involvement in relevant pathways. As reported in Figure 4, the DE of all selected genes has

-20 -

Animal Genetics 
457 been validated, with high correlation between the fold changes obtained by RNA-seq and by 458 qRT-PCR data.

460 DE transcript characterisation

461 We characterized the cDEG in terms of their functional role in adipose tissue. Using DAVID

462 Bioinformatics Resources we first identified the functional categories, enriched in genes

463 differentially regulated between FAT and LEAN groups.

464 The Biological Process categories enriched in higher expressed DEG are response to

465 stimulus, immune system process and cell activation, skeletal system development (Table

466 3). DAVID clustering of the few lower expressed genes detected (ADSSL1, CDO1, DNAJB1,

467 HSPA1A, HSPA1B, HSPA2, HSPB8, IGFBP5, OLFML3) allowed to identify the functional

468 categories unfolded protein binding and stress response represented by five heat shock

469 protein genes that are involved in protein stabilization after cellular stress.

470 Apart from the Gene Ontology-based functional characterization of the whole subsets of

471 higher- and lower-expressed genes we considered cDEG function and involvement in

472 specific pathways, according to literature and knowledge bases.

473 Several more expressed genes in FAT animals ( $A C P 5, B C L 2 A 1, C D 1 A, E G R 2, E N P P 1, G P N M B$,

474 INHBB, LYZ, MSR1, OLR1, PIK3AP1, PLIN2, SPP1, STC1) are characterized by a metabolic

475 function mainly related to adipocyte growth regulation, while others (CCR1, CD163,

476 SLC11A1) are known to be involved in immune defence of the organism.

478 Discussion

479 Transcriptome data highlight the adipose tissue complexity

$-21-$ 
480 The deep sequencing analysis of pig backfat transcriptome performed allowed finding

481 thousands of genes and transcripts expressed. In the present study, we applied stringent

482 cleaning and filtering procedures of the sequencing data and, on average, 90 million reads

483 per sample were mapped, obtaining a higher sequencing depth compared to previous

484 studies (Chen et al., 2011; Jiang et al., 2013; Sodhi et al., 2014; Wang et al., 2014).

485 The adipose tissue is not only metabolically and transcriptionally active, but has been

486 recognized as an important endocrine organ (Kershaw et al., 2004; Trayhurn et al., 2005).

487 Adipocytes are a dynamic and highly regulated population of cells (Rosen \& MacDougald,

488 2006; Moreno-Navarrete \& Fernández-Real, 2012). Our results agree with these data

489 supporting the characterization of the adipocytes as highly specialised endocrine cells that

490 can play key roles in various physiological processes. The multifunctionality and the

491 complexity of the tissue is witnessed also by the high number of transcripts (more than sixty

492 thousands) found in the present study, including many new transcripts from previously non-

493 annotated loci in porcine genome. The majority of the reconstructed sequences are novel

494 isoforms of already known genes that express more than two different transcripts each.

495 Similar patterns observed in human cells (Djebali et al., 2012) and the high quality of the

496 sequenced reads used in our analysis support the idea that this is more attributable to an

497 incomplete annotation of the transcript isoforms expressed in pig backfat, than to transcript

498 reconstruction artefacts. The different isoforms derived from the same locus arisen from

499 our analysis and observed for almost half of the expressed genes, may contribute to

500 improve the knowledge of the porcine transcriptome, and to refine the current swine

501 genome annotation. The new PLIN2 isoforms reported above are an interesting example,

502 especially if compared to the human genome where at least eight PLIN2 transcript isoforms

503 are annotated and only four of them are coding. Remarkably, three human PLIN2 isoforms

$$
-22-
$$


504 encode $\mathrm{N}$-terminal truncated amino acid chains that are similar to the truncated isoform we

505 reconstructed in our study, and whose function has not yet been elucidated. Furthermore,

506 Russell et al. (2008) identified in a PLIN2 deficient mouse cell line the expression of a PLIN2

507 C-terminal truncated protein that may partially replace the function of the full-length

508 protein. Additional studies are needed to understand if and how the short transcript we

509 found differentially expressed could change the gene functions compared to the wild type

510 long protein.

511

512 Functional characterisation of the adipose tissue expression profile

513 The profile of the subcutaneous adipose tissue transcriptome in pigs was delineated and the

514 functional analysis of the genes expressed in backfat tissue was performed to know their

515 metabolic role and to connect them to specific competences of the tissue. We didn't find

516 particular differences between the functional categories of the genes expressed in the

517 backfat tissue of FAT and LEAN pigs. More in details among the most expressed genes in the

518 fat tissue, many are involved in metabolic pathways and biological processes related to

519 protein metabolism, oxidoreductase activity for ATP production, regulation of lipid synthesis

520 and degradation.

521

522 Genes differentially expressed between LEAN and FAT animals converge and

523 connect to specific functions

524 The detection of DE genes and transcripts has been obtained by a stringent procedure

525 grounding on integration of different methods for expression estimation and differential

526 expression testing, as done in a recent study (Ropka-Molik et al., 2014) focused to muscle

$$
-23-
$$


527 tissue gene expression in pigs of different breeds. In the present study, which compares pigs

528 of the same breed and reared under standard conditions, we detected significant gene

529 expression variations. The sensitivity of our approach was supported by the successful

530 validation of all the eleven DEG assayed.

531 We analyzed the biological functions of genes differentially expressed between FAT and

532 LEAN animals (Figure 5). It is interesting to note that the main differences were found for

533 functional categories of genes related Inflammation and immunity that resulted more

534 expressed in FAT pigs. The genes less expressed in FAT animals include some heat shock

535 protein genes. The biological functions of DEGs show a stronger activation in adipose tissue

536 of FAT pigs of genes for important processes involved in hypertrophy and adipogenesis, such

537 as differentiation and maturation. Supposedly, these biological processes could be altered in

538 adipose tissue of FAT pigs due to dysregulated adipose metabolism and endocrinology

539 similarly to what was hypothesized in humans (Sethi, 2010). On the whole, there is a

540 consistent difference concerning the biological functions characterizing the most expressed

541 genes on backfat tissue and those of the genes differentially expressed between FAT and

542 LEAN pigs.

544 Some genes higher expressed in FAT animals could modulate backfat

545 physiological processes

546 Specific DEGs more expressed in FAT pigs participate to biochemical pathways related to

547 and involved in adipocytes metabolism and adipose tissue physiology. Ectonucleotide

548 pyrophosphatase/phosphodiesterase 1 (ENPP1) encodes a catalytic enzyme involved in

549 adipocyte maturation (Liang et al., 2007). Pan et al. (2011) showed that the over-expression

$-24-$ 
550 of ENPP1 in a human cell line resulted in adipocyte insulin resistance and demonstrated an

551 association with fatty liver, hyperlipidemia, and dysglycemia. Accordingly, the study of

552 Chandalia et al. (2012) underlined an increased ENPP1 expression in adipose tissue

553 associated with defective adipocyte maturation leading to pathogenesis of insulin resistance

554 and its associated complications for glucose and lipid metabolism in absence of obesity. In

555 addition, Meyre et al. (2005) reported the presence of three ENPP1 SNPs in human gene

556 associated with adult obesity and increased risk of glucose intolerance and type 2 diabetes.

557 Furthermore, also the genes acid phosphatase 5, tartrate resistant (ACP5) and lysozyme

$558(L Y Z)$ that in this research have higher transcriptional level in FAT pigs have been reported to

559 be involved in excessive backfat deposition in pigs and in the development of

560 atherosclerosis (Padilla et al., 2013).

561 In the present research, some genes overexpressed in the adipose tissue of FAT pigs, namely

$562 S T C 1, E G R 2$, and INHBB, are related to adipocyte differentiation and adipocyte maturation.

563 STC1 (Stanniocalcin 1) has been reported in literature to be up-regulated during

564 adipogenesis and to modulate steroidogenesis. Serlachius \& Andersson (2004) related STC1

565 up-regulation to the set of survival genes in adipocyte differentiation, which is also

566 associated to overexpression of the anti-apoptotic proteins BCL2 reported to be involved in

567 inflammation pathway. EGR2 (early growth response 2) is a direct target of mir-224-5p, a

568 negative regulator of adipocyte differentiation that is down regulated during the early

569 process of mouse adipocyte differentiation, and the expression of EGR2 is increased (Peng

570 et al., 2013). The INHBB (Inhibin beta B) gene coding for the activin B subunit is part of the

571 inhibins/activins family of proteins with cytokine and hormone activity. In human and mice,

572 INHBB has been associated to the physiological and metabolic modifications during

573 adipogenesis when it is highly expressed and is the predominant activin in human adipose

$-25-$ 
574 tissue (Hoggard et al., 2009). INHBB is member of TGF-protein superfamily of secreted

575 growth factors involved in many biological responses including regulation of apoptosis;

576 proliferation and differentiation of human adipocytes; tissue remodeling; and inflammatory

577 immune response (Dani C., 2013). It can be hypothesized that in FAT pigs the pro-adipogenic

578 INHBB gene expression increases as it is involved in the differentiation of preadipocytes into

579 mature adipocyte, and that INHBB is involved in many physiological processes and including

580 the control of food intake and to energy metabolism through the regulation of hypothalamic

581 and pituitary hormone secretions. Another gene overexpressed in FAT pigs related to

582 feeding and pituitary secretions is GPNMB (glycoprotein transmembrane NMB). GPNMB is

583 one of the receptors activated by bombesin-like endogenous peptide ligands, such as

584 gastrin-releasing peptide (GRP), neuromedin B (NMB) and neuromedin C (GRP18-27). These

585 receptors are involved in the regulation of many biological functions including

586 thermoregulation, feeding, pituitary, gastric and pancreatic secretion. The NMB/NMB-R

587 pathway is involved in the regulation of a wide variety of behaviours, such as spontaneous

588 activity, feeding, and anxiety-related behaviour (Yamada et al., 2002).

589 The OLR1 (Oxidized low density lipoprotein (lectin-like) receptor 1) gene resulted more

590 expressed in FAT pigs compared to LEAN animals. This gene codes for a LDL receptor that

591 belongs to the C-type lectin superfamily, one of many target genes, including perilipins, of

592 the PPAR signalling, which is involved specifically in lipid metabolism and fatty acids

593 transport. In this way, $O L R 1$ is a receptor that mediates the recognition, internalization and

594 degradation of oxidatively modified low-density lipoprotein by vascular endothelial cells.

595 OLR1 removes oxidised low-density lipoproteins from the circulation, as part of lipid

596 metabolism pathways (Mehta et al., 2002). 
598 Genes involved in immunity and inflammation are more expressed in FAT

599 animals

600 Some other genes overexpressed in FAT pigs are related to immunity. Inflammatory links

601 between human obesity and metabolic diseases are well known mechanisms based on the

602 recruitment of immune cells into adipose tissue (Kabir et al., 2014). The development of a

603 pre-inflammatory condition in presence of dysregulated excessive adipogenesis is

604 associated with adipose macrophage infiltration and activation. From our study, we can

605 hypothesize a similar process in backfat tissue of FAT pigs where we identified the over

606 expression of the gene macrophage scavenger receptor 1 (MSR1), a membrane glycoprotein

607 that in humans is involved in the pathologic deposition of cholesterol in arterial walls during

608 atherogenesis (Haasken et al., 2013). Additionally, the overexpression of secreted

609 phosphoprotein 1 (SPP1) in FAT pigs can suggest the hypothesis that this gene is acting as a

610 proinflammatory cytokine that promotes monocyte chemotaxis and cell motility and might

611 link, in pigs like in mice, fat accumulation to the development of insulin resistance by

612 sustaining inflammation and the accumulation of macrophages in adipose tissue (Nomiyana

613 et al. 2007). Interestingly, a porcine SPP1 gene polymorphism was associated to backfat

614 thickness in the Landrace $\times$ Jeju (Korea) Black pig F2 population (Han et al., 2012). SPP1

615 might play a key role in the pathway that leads to type I immunity enhancing interferon-

616 gamma and interleukin-12 production and suppressing interleukin-10 (Ashkar et al., 2000).

617 Therefore, these data allow hypothesizing SPP1 as a gene associated, in pigs like to in human,

618 to the link between obesity, adipose tissue inflammation, and insulin resistance. In addition,

619 phosphoinositide-3-kinase adaptor protein 1 (PIK3AP1), higher expressed in FAT pigs, is a positive regulator of phosphatidylinositol 3-kinase $(P I 3 K)$ signalling. $P I 3 K$ signalling pathway

$-27-$ 
621 has a key role in the insulin-dependent regulation of adipocyte metabolism (glucose and

622 lipid metabolism). Besides, PI3K participate in obesity-associated inflammatory cell

623 recruitment (neutrophils and macrophages), as well as in the CNS-dependent neurohumoral

624 regulation of food intake/energy expenditure (McCurdy \& Clemm, 2013; Beretta et al., 625 2015).

626 Other genes found in the present research and related to inflammatory condition of the 627 adipose tissue in FAT pigs are particularly interesting to mention. CD163, member of the 628 scavenger receptor cysteine-rich superfamily (Guo et al., 2014; Smith et al., 2014); solute 629 carrier family 11 (proton-coupled divalent metal ion transporter), member 1 (SLC11A1), a 630 gene involved in the resistance to Salmonella infection (Kommadath et al., 2014) as well as 631 the chemokine ( $C-C$ motif) receptor 1 (CCR1), that was previously found overexpressed in 632 obese pigs (Kogelman et al., 2014); BCL2-related protein A1 (BCL2A1), a gene found to be 633 overexpressed in pigs with an high obesity index and that is related to immunity, 634 inflammatory pathway, and osteoclast differentiation (Kogelman et al., 2014); CD1a 635 molecule ( $C D 1 A$, indicated as $P C D 1 A$ on the cited paper), a surface antigen involved in 636 immunity was found to be overexpressed in obese pigs by Kogelman et al. (2014). The same

637 Authors highlighted a strong connection between fat deposition on the body (obesity),

638 immunity and bone development. They also indicated that CCR1 gene is a strong candidate

639 regulator of immune response as it is a receptor of pro-inflammatory chemokines in adipose

640 tissue playing a pivotal role in obesity-associated diseases (Kabir et al. 2014; Lumeng \&

641 Saltiel, 2011).

642

643 Heat shock response, protein folding and repair are impaired in FAT animals

$-28-$ 
644 Considering the 14 genes less expressed in FAT animals, direct relationships with lipid

645 metabolism are not apparent. However, the "unfolded protein binding" function is enriched

646 among these genes, which include five functionally linked heat shock proteins (DNAJB1,

647 HSPA1A, HSPA1B, HSPA2 and HSPB8). Heat shock proteins are involved in stabilization of

648 existing proteins against aggregation, mediating the folding of newly translated proteins in

649 the cytosol and in organelles, and also in the ubiquitin-proteasome pathway. DNAJB1, a

650 member of the Hsp40 family, is a molecular chaperon involved in protein folding and

651 protein complex assembly. DNAJB1, a member of the Hsp40 family, promotes protein

652 folding and prevent misfolded protein aggregation, as HSPB8, a member of the Hsp20

653 family, does (Vicario et al., 2014). DNAJB1 also stimulates the ATPase activity of protein of

654 the Hsp70 family to which other genes less expressed in FAT pigs (HSPA1A, HSPA1B, and

655 HSPA2) belong, indicating a possible functional link between these four genes. Our results

656 suggest a general impairment of the protein folding and repair in the fattest animals, in

657 accordance to previous observations of studies carried out on human obesity. Obesity is a

658 pathological human condition in which a chronically positive energy balance induces in

659 adipocytes, the cells in charge to store the excess of energy in fat depots, a persistent stress

660 activating in turn defence processes as autophagy or apoptosis.

661 As reviewed by Newsholme \& de Bittencourt (2014), if the heat shock response, a key

662 component of the physiological response to resolve inflammation, is hampered in adipose

663 tissue, the adipocyte metabolic stress triggers fat cell senescence with reduction of the heat

664 shock proteins activity. In this condition, the advance of inflammasome mediated secretory

665 activity from adipose to other tissues promotes cellular senescence in many other cells of

666 the organism, aggravating obesity-dependent chronic inflammation. This mechanism could

667 have been activated also in the FAT pigs of our experiment (Figure 5) due to a genetic

$-29-$ 
668 aptitude of the fattest animals toward a higher fat deposition and adiposity similar to

669 obesity. Indeed, a decrease in the synthesis of the mRNAs of the heat shock proteins and an

670 increase of the expression of many genes related to an inflammatory status and to immune

671 response is a characteristic of the fattest pigs. Increase of the expression of INHBB and SPP1

672 denotes for instance the augmented production of cytokines and the higher expression of

673 ENPP1 and PIK3AP1 may indicate a status of insulin resistance, one of the typical signals

674 connected with obesity.

675

676 Pig backfat deposition and impaired stress response may activate inflammation

677 Our results agree with recent studies showing that several immune system and anti-

678 inflammatory processes are activated and play a critical role in the response to fat

679 accumulation in porcine backfat tissue (Sodhi et al., 2014) and in visceral fat tissue

680 (Toedebusch et al., 2014; Wang et al., 2014). Wang et al. (2014) and Zhou et al. (2013) used

681 three female Landrace pigs to identify DEG between subcutaneous, visceral and

682 intramuscular fat indicating that visceral and intramuscular adipose tissues were mainly

683 associated with inflammatory features of the tissue and immune response. Our data suggest

684 that also in backfat a predominant role of immunity processes is related to an increased

685 adipose tissue deposition.

686 The results obtained seem to sustain the hypothesis that the high fat accumulation in

687 adipose tissue of pigs can determine the development of an inflammatory process

688 producing a cascade of defence and adaptive reactions in the tissue, such as activation of

689 immune system and mesenchymal cells differentiation in adipocytes.

$-30-$ 
690 A deeper knowledge of the metabolic processes involved in fat deposition can be very

691 important to develop the use the pig as model species to study obesity and related

692 disorders for humans because of similar anatomy and physiology (Spurlock \& Gabler, 2008;

693 Litten-Brown et al., 2010; Varga et al., 2010) and considering the above described

694 similarities between pigs and humans.

695 In order to fully elucidate the complex gene network regulating backfat deposition on pigs,

696 it will be important to extend the basic knowledge by further coding and non-coding

697 transcriptome characterization. Additional information would probably come from studying

698 interactions between the differentially expressed long RNAs identified in the present paper

699 and the regulatory microRNAs expressed in porcine adipose tissue identified on some of the 700 same animals (Gaffo et al., 2014).

701 The results of the present work unlock the opportunity that some of the identified

702 differentially expressed genes might be used as biomarkers (Ibáñez-Escriche et al., 2014) to 703 improve carcass fat traits in to look for SNPs regulating their expression to be included in 704 selection schemes to make more sustainable the pig production chain.

705

706 Acknowledgements

707 We thank Dr. Maurizio Gallo from Associazione Nazionale Allevatori Suini (ANAS) and Dr.

708 Luca Buttazzoni (CREA-PCM) for providing the porcine backfat tissue samples, BMR

709 genomics for performing the RNA-Seq. This work was supported by Progetto "AGER -

710 Agroalimentare e ricerca": Advanced research in genomics and processing technologies for

711 the Italian heavy pig production - Hepiget (Grant N. 2011- 0279).

712

$-31-$ 


\section{References}

714 Anders, S., \& Huber, W. (2010) Differential expression analysis for sequence count data.

$715 \quad$ Genome Biol. 11, R106.

716 Anders, S., Pyl, P.T. \& Huber, W. (2015) HTSeq--a Python framework to work with high-

717 throughput sequencing data. Bioinformatics 31, 166-169.

718 Ashkar, S., Weber, G.F., Panoutsakopoulou, V., Sanchirico, M.E., Jansson, M., Zawaideh, S.,

719 Rittling, S.R., Denhardt, D.T., Glimcher, M.J. \& Cantor, H. (2000) Eta-1 (osteopontin): an

720 early component of type-1 (cell-mediated) immunity. Science $287,860-864$.

721 Beretta, M., Bauer, M. \& Hirsch, E. (2015) PI3K signaling in the pathogenesis of obesity: The

722 cause and the cure. Adv Biol Regul. 58, 1-15.

723 Bosi, P. \& Russo, V. (2004) The production of the heavy pig for high quality processed

724 products. Italian Journal of Animal Science 3, 309-321.

725 Bustin, S.A. \& Nolan, T. (2004) Pitfalls of quantitative real-time reverse-transcription

726 polymerase chain reaction. J Biomol Tech 15, 155-166.

727 Cabili, M.N., Trapnell, C., Goff, L., Koziol, M., Tazon-Vega, B., Regev, A. \& Rinn, J.L. (2011)

728 Integrative annotation of human large intergenic noncoding RNAs reveals global

729 properties and specific subclasses. Genes Dev. 25, 1915-1927.

730 Čandek-Potokar, M. \& Škrlep, M. (2012) Factors in pig production that impact the quality of

731 dry-cured ham: a review. Animal 6, 327-338.

732 Chandalia, M., Davila, H., Pan, W., Szuszkiewicz, M., Tuvdendorj, D., Livingston, E.H. \&

733 Abate, N. (2012) Adipose tissue dysfunction in humans: a potential role for the

734 transmembrane protein ENPP1. J. Clin. Endocrinol. Metab. 97, 4663-4672.

$-32-$ 
735 Chen, C., Ai, H., Ren, J., Li, W., Li, P., Qiao, R., Ouyang, J., Yang, M., Ma, J. \& Huang, L. (2011)

736 A global view of porcine transcriptome in three tissues from a full-sib pair with extreme

737 phenotypes in growth and fat deposition by paired-end RNA sequencing. BMC Genomics

$738 \quad 12,448$.

739 Chen, C.H., Lin, E.C., Cheng, W.T.K., Sun, H.S., Mersmann, H.J. \& Ding, S.T. (2006) Abundantly

740 expressed genes in pig adipose tissue: an expressed sequence tag approach. J. Anim. Sci

$741 \quad 84,2673-2683$.

742 Conte, M., Vasuri, F., Trisolino, G., Bellavista, E., Santoro, A., Degiovanni, A., Martucci, E.,

743 D’Errico-Grigioni, A., Caporossi, D., Capri, M., Maier, A.B., Seynnes, O., Barberi, L.,

744 Musarò, A., Narici, M.V., Franceschi, C. \& Salvioli, S. (2013) Increased Plin2 expression in

745 human skeletal muscle is associated with sarcopenia and muscle weakness. PLoS ONE 8 ,

$746 \quad$ e73709.

747 Corominas, J., Ramayo-Caldas, Y., Puig-Oliveras, A., Estellé, J., Castelló, A., Alves, E., Pena,

748 R.N., Ballester, M. \& Folch, J.M. (2013) Analysis of porcine adipose tissue transcriptome

749 reveals differences in de novo fatty acid synthesis in pigs with divergent muscle fatty

$750 \quad$ acid composition. BMC Genomics $14,843$.

751 Cox, M.P., Peterson, D.A. \& Biggs, P.J. (2010) SolexaQA: At-a-glance quality assessment of

752 Illumina second-generation sequencing data. BMC Bioinformatics 11, 485.

753 Dani, C. (2013) Activins in adipogenesis and obesity. Int J Obes (Lond) 37, 163-166.

754 Davoli, R., Gandolfi, G., Braglia, S., Comella, M., Zambonelli, P., Buttazzoni, L. \& Russo, V.

755 (2010) New SNP of the porcine Perilipin 2 (PLIN2) gene, association with carcass traits

756 and expression analysis in skeletal muscle. Mol Biol Rep 38, 1575-1583.

$-33-$ 
757 Durinck, S., Spellman, P.T., Birney, E. \& Huber, W. (2009) Mapping identifiers for the

758 integration of genomic datasets with the R/Bioconductor package biomaRt. Nat Protoc

$759 \quad 4,1184-1191$.

760 Djebali, S., Davis, C.A., Merkel, A., Dobin, A., Lassmann, T., Mortazavi, A.M., Tanzer, A.,

761 Lagarde, J., Lin, W., Schlesinger, F., Xue, C., Marinov, G.K., Khatun, J., Williams, B.A.,

762 Zaleski, C., Rozowsky, J., Röder, M., Kokocinski, F., Abdelhamid, R.F., Alioto, T.,

763 Antoshechkin, I., Baer, M.T., Bar, N.S., Batut, P., Bell, K., Bell, I., Chakrabortty, S., Chen,

764 X., Chrast, J., Curado, J., Derrien, T., Drenkow, J., Dumais, E., Dumais, J., Duttagupta, R.,

765 Falconnet, E., Fastuca, M., Fejes-Toth, K., Ferreira, P., Foissac, S., Fullwood, M.J., Gao, H.,

766 Gonzalez, D., Gordon, A., Gunawardena, H., Howald, C., Jha, S., Johnson, R., Kapranov,

767 P., King, B., Kingswood, C., Luo, O.J., Park, E., Persaud, K., Preall, J.B., Ribeca, P., Risk, B.,

768 Robyr, D., Sammeth, M., Schaffer, L., See, L.-H., Shahab, A., Skancke, J., Suzuki, A.M.,

769 Takahashi, H., Tilgner, H., Trout, D., Walters, N., Wang, H., Wrobel, J., Yu, Y., Ruan, X.,

770 Hayashizaki, Y., Harrow, J., Gerstein, M., Hubbard, T., Reymond, A., Antonarakis, S.E.,

771 Hannon, G., Giddings, M.C., Ruan, Y., Wold, B., Carninci, P., Guigó, R., Gingeras, T.R.,

772 (2012) Landscape of transcription in human cells. Nature 489, 101-108.

773 Ferraz, A.L.J., Ojeda, A., López-Béjar, M., Fernandes, L.T., Castelló, A., Folch, J.M. \& Pérez-

774 Enciso, M. (2008) Transcriptome architecture across tissues in the pig. BMC Genomics 9, $775 \quad 173$.

776 Gaffo, E., Zambonelli, P., Bisognin, A., Bortoluzzi, S. \& Davoli, R. (2014) miRNome of Italian

777 Large White pig subcutaneous fat tissue: new miRNAs, isomiRs and moRNAs. Anim.

$778 \quad$ Genet. 45, 685-698.

779 Gandolfi, G., Mazzoni, M., Zambonelli, P., Lalatta-Costerbosa, G., Tronca, A., Russo, V. \&

780 Davoli, R. (2011) Perilipin 1 and perilipin 2 protein localization and gene expression study $-34-$ 
781 in skeletal muscles of European cross-breed pigs with different intramuscular fat 782 contents. Meat Sci 88, 631-637.

783 Gorodkin, J., Cirera, S., Hedegaard, J., Gilchrist, M.J., Panitz, F., Jørgensen, C., Scheibye-

784 Knudsen, K., Arvin, T., Lumholdt, S., Sawera, M., Green, T., Nielsen, B.J., Havgaard, J.H.,

785 Rosenkilde, C., Wang, J., Li, H., Li, R., Liu, B., Hu, S., Dong, W., Li, W., Yu, J., Wang, J.,

786 Stærfeldt, H.-H., Wernersson, R., Madsen, L.B., Thomsen, B., Hornshøj, H., Bujie, Z.,

787 Wang, X., Wang, X., Bolund, L., Brunak, S., Yang, H., Bendixen, C. \& Fredholm, M. (2007)

788 Porcine transcriptome analysis based on 97 non-normalized cDNA libraries and assembly

789 of $1,021,891$ expressed sequence tags. Genome Biology 8, R45.

790 Guo, L., Niu, J., Yu, H., Gu, W., Li, R., Luo, X., Huang, M., Tian, Z., Feng, L. \& Wang, Y. (2014)

791 Modulation of CD163 expression by metalloprotease ADAM17 regulates porcine

792 reproductive and respiratory syndrome virus entry. J. Virol. 88, 10448-10458.

793 Haasken, S., Auger, J.L., Taylor, J.J., Hobday, P.M., Goudy, B.D., Titcombe, P.J., Mueller, D.L.

794 \& Binstadt, B.A. (2013) Macrophage scavenger receptor 1 (Msr1, SR-A) influences B cell

795 autoimmunity by regulating soluble autoantigen concentration. J. Immunol. 191, 1055-

7961062.

797 Han, S.-H., Shin, K.-Y., Lee, S.-S., Ko, M.-S., Oh, H.-S. \& Cho, I.-C. (2012) Porcine SPP1 gene

798 polymorphism association with phenotypic traits in the Landrace $\times$ Jeju (Korea) Black pig

$799 \quad$ F2 population. Mol. Biol. Rep. 39, 7705-7709.

800 Henderson, C.R. \& Quaas, R.L. (1976) Multiple Trait Evaluation Using Relatives' Records.

$801 \quad$ Journal of Animal Science 43, 1188-1197.

802 Hoggard, N., Cruickshank, M., Moar, K.M., Barrett, P., Bashir, S. \& Miller, J.D.B. (2009)

803 Inhibin betaB expression in murine adipose tissue and its regulation by leptin, insulin

804 and dexamethasone. J. Mol. Endocrinol. 43, 171-177.

$-35-$ 
805 Hornshøj, H., Conley, L.N., Hedegaard, J., Sørensen, P., Panitz, F. \& Bendixen, C. (2007)

806 Microarray Expression Profiles of 20.000 Genes across 23 Healthy Porcine Tissues. PLoS

807 ONE 2, e1203.

808 Huang, D.W., Sherman, B.T. \& Lempicki, R.A. (2009) Systematic and integrative analysis of

809 large gene lists using DAVID bioinformatics resources. Nat Protoc 4, 44-57.

810 Ibáñez-Escriche, N., Forni, S., Noguera, J.L. \& Varona, L. (2014) Genomic information in pig

811 breeding: Science meets industry needs. Livestock Science 166, 94-100.

812 Iyer, M.K., Niknafs, Y.S., Malik, R., Singhal, U., Sahu, A., Hosono, Y., Barrette, T.R., Prensner,

813 J.R., Evans, J.R., Zhao, S., Poliakov, A., Cao, X., Dhanasekaran, S.M., Wu, Y.-M., Robinson,

814 D.R., Beer, D.G., Feng, F.Y., Iyer, H.K. \& Chinnaiyan, A.M. (2015) The landscape of long

815 noncoding RNAs in the human transcriptome. Nat. Genet. 47, 199-208.

816 Jiang, S., Wei, H., Song, T., Yang, Y., Peng, J. \& Jiang, S. (2013) Transcriptome Comparison

817 between Porcine Subcutaneous and Intramuscular Stromal Vascular Cells during

818 Adipogenic Differentiation. PLoS ONE 8, e77094.

819 Kabir, S.M., Lee, E.-S. \& Son, D.-S. (2014) Chemokine network during adipogenesis in 3T3-L1

820 cells: Differential response between growth and proinflammatory factor in

821 preadipocytes vs. adipocytes. Adipocyte 3, 97-106.

822 Kershaw, E.E. \& Flier, J.S. (2004) Adipose tissue as an endocrine organ. J. Clin. Endocrinol.

$823 \quad$ Metab. 89, 2548-2556.

824 Kim, D., Pertea, G., Trapnell, C., Pimentel, H., Kelley, R. \& Salzberg, S.L. (2013) TopHat2:

825 accurate alignment of transcriptomes in the presence of insertions, deletions and gene

826 fusions. Genome Biol. 14, R36.

827 Kogelman, L.J.A., Cirera, S., Zhernakova, D.V., Fredholm, M., Franke, L. \& Kadarmideen, H.N.

828 (2014) Identification of co-expression gene networks, regulatory genes and pathways for $-36-$ 
829 obesity based on adipose tissue RNA Sequencing in a porcine model. BMC Med

$830 \quad$ Genomics 7, 57.

831 Kommadath, A., Bao, H., Arantes, A.S., Plastow, G.S., Tuggle, C.K., Bearson, S.M.D., Guan,

832 L.L. \& Stothard, P. (2014) Gene co-expression network analysis identifies porcine genes

833 associated with variation in Salmonella shedding. BMC Genomics 15, 452.

834 Kong, L., Zhang, Y., Ye, Z.-Q., Liu, X.-Q., Zhao, S.-Q., Wei, L. \& Gao, G. (2007) CPC: assess the

835 protein-coding potential of transcripts using sequence features and support vector

836 machine. Nucleic Acids Res. 35, W345-349.

837 Langmead, B. \& Salzberg, S.L. (2012) Fast gapped-read alignment with Bowtie 2. Nat.

$838 \quad$ Methods 9, 357-359.

839 Li, H., Handsaker, B., Wysoker, A., Fennell, T., Ruan, J., Homer, N., Marth, G., Abecasis, G. \&

840 Durbin, R. (2009) 1000 Genome Project Data Processing Subgroup. The Sequence

841 Alignment/Map format and SAMtools. Bioinformatics 25, 2078-2079.

842 Li, X.J., Yang, H., Li, G.X., Zhang, G.H., Cheng, J., Guan, H. \& Yang, G.S. (2012) Transcriptome

843 profile analysis of porcine adipose tissue by high-throughput sequencing. Animal

$844 \quad$ Genetics 43, 144-152.

845 Liang, J., Fu, M., Ciociola, E., Chandalia, M. \& Abate, N. (2007) Role of ENPP1 on Adipocyte

$846 \quad$ Maturation. PLoS ONE 2, e882.

847 Litten-Brown, J.C., Corson, A.M. \& Clarke, L. (2010) Porcine models for the metabolic

848 syndrome, digestive and bone disorders: a general overview. Animal 4, 899-920.

849 Lumeng, C.N. \& Saltiel, A.R. (2011) Inflammatory links between obesity and metabolic

$850 \quad$ disease. J. Clin. Invest. 121, 2111-2117.

851 McCurdy, C.E. \& Klemm, D.J. (2013) Adipose tissue insulin sensitivity and macrophage

852 recruitment: Does PI3K pick the pathway? Adipocyte 2, 135-142.

$$
-37-
$$


853 Mehta, J.L. \& Li, D. (2002) Identification, regulation and function of a novel lectin-like

854 oxidized low-density lipoprotein receptor. J. Am. Coll. Cardiol. 39, 1429-1435.

855 Meyre, D., Bouatia-Naji, N., Tounian, A., Samson, C., Lecoeur, C., Vatin, V., Ghoussaini, M.,

856 Wachter, C., Hercberg, S., Charpentier, G., Patsch, W., Pattou, F., Charles, M.-A.,

857 Tounian, P., Clément, K., Jouret, B., Weill, J., Maddux, B.A., Goldfine, I.D., Walley, A.,

858 Boutin, P., Dina, C. \& Froguel, P. (2005) Variants of ENPP1 are associated with childhood

859 and adult obesity and increase the risk of glucose intolerance and type 2 diabetes. Nat

$860 \quad$ Genet 37, 863-867.

861 Mikawa, A., Suzuki, H., Suzuki, K., Toki, D., Uenishi, H., Awata, T. \& Hamasima, N. (2004)

862 Characterization of 298 ESTs from porcine back fat tissue and their assignment to the

863 SSRH radiation hybrid map. Mamm Genome 15, 315-322.

864 Moon, J.-K., Kim, K.-S., Kim, J.-J., Choi, B.-H., Cho, B.-W., Kim, T.-H. \& Lee, C.-K. (2009)

865 Differentially expressed transcripts in adipose tissue between Korean native pig and

866 Yorkshire breeds. Anim. Genet. 40, 115-118.

867 Moreno-Navarrete, J.M., \& Fernández-Real, J.M. (2012) Adipocyte Differentiation. In

868 Adipose Tissue Biology (ed. by M.E. Symonds). pp. 17-38. Springer, New York

869 Morgulis, A., Coulouris, G., Raytselis, Y., Madden, T.L., Agarwala, R. \& Schäffer, A.A. (2008)

870 Database indexing for production MegaBLAST searches. Bioinformatics 24, 1757-1764.

871 Newsholme, P. \& de Bittencourt, P.I.H. (2014) The fat cell senescence hypothesis: a

872 mechanism responsible for abrogating the resolution of inflammation in chronic disease.

873 Current Opinion in Clinical Nutrition and Metabolic Care 17, 295-305.

874 Padilla, J., Jenkins, N.T., Lee, S., Zhang, H., Cui, J., Zuidema, M.Y., Zhang, C., Hill, M.A.,

875 Perfield, J.W., Ibdah, J.A., Booth, F.W., Davis, J.W., Laughlin, M.H. \& Rector, R.S. (2013)

$-38-$ 
876 Vascular transcriptional alterations produced by juvenile obesity in Ossabaw swine.

877 Physiol. Genomics 45, 434-446.

878 Pan, W., Ciociola, E., Saraf, M., Tumurbaatar, B., Tuvdendorj, D., Prasad, S., Chandalia, M. \&

879 Abate, N. (2011) Metabolic consequences of ENPP1 overexpression in adipose tissue.

$880 \quad$ Am. J. Physiol. Endocrinol. Metab. 301, E901-911.

881 Peng, Y., Xiang, H., Chen, C., Zheng, R., Chai, J., Peng, J. \& Jiang, S. (2013) MiR-224 impairs

882 adipocyte early differentiation and regulates fatty acid metabolism. The International

883 Journal of Biochemistry \& Cell Biology 45, 1585-1593.

884 Quinlan, A.R. \& Hall, I.M. (2010) BEDTools: a flexible suite of utilities for comparing genomic 885 features. Bioinformatics 26, 841-842.

886 Roberts, A., Pimentel, H., Trapnell, C. \& Pachter, L. (2011a) Identification of novel transcripts

887 in annotated genomes using RNA-Seq. Bioinformatics 27, 2325-2329.

888 Roberts, A., Trapnell, C., Donaghey, J., Rinn, J.L. \& Pachter, L. (2011b) Improving RNA-Seq

889 expression estimates by correcting for fragment bias. Genome Biol. 12, R22.

890 Ropka-Molik, K., Zukowski, K., Eckert, R., Gurgul, A., Piórkowska, K. \& Oczkowicz, M. (2014)

891 Comprehensive analysis of the whole transcriptomes from two different pig breeds

892 using RNA-Seq method. Anim. Genet. 45, 674-684.

893 Rosen, E.D. \& MacDougald, O.A. (2006) Adipocyte differentiation from the inside out. Nat.

$894 \quad$ Rev. Mol. Cell Biol. 7, 885-896.

895 Russell, T.D., Palmer, C.A., Orlicky, D.J., Bales, E.S., Chang, B.H.-J., Chan, L. \& McManaman, 896 J.L. (2008) Mammary glands of adipophilin-null mice produce an amino-terminally 897 truncated form of adipophilin that mediates milk lipid droplet formation and secretion. $898 \quad$ J. Lipid Res. 49, 206-216.

$-39-$ 
899 Russo, V., Buttazzoni, L., Baiocco, C., Davoli, M.R., Nanni Costa, N.L., Schivazappa, O.C. \&

900 Virgili, P.C. (2000) Heritability of muscular cathepsin B activity in Italian Large White pigs.

$901 \quad J o u r n a l$ of Animal Breeding and Genetics 117, 37-42.

902 Russo, V., Fontanesi, L., Scotti, E., Beretti, F., Davoli, R., Nanni Costa, L., Virgili, R. \&

903 Buttazzoni, L. (2008) Single nucleotide polymorphisms in several porcine cathepsin

904 genes are associated with growth, carcass, and production traits in Italian Large White

905 pigs. Journal of Animal Science 86, 3300-3314.

906 Serlachius, M. \& Andersson, L.C. (2004) Upregulated expression of stanniocalcin-1 during

907 adipogenesis. Exp. Cell Res. 296, 256-264.Sethi, J.K. (2010) Activatin' human adipose

908 progenitors in obesity. Diabetes 59, 2354-2357.

909 Sethi, J.K. (2010) Activatin' Human Adipose Progenitors in Obesity. Diabetes 59, 2354-2357.

910 Smith, S.H., Wilson, A.D., Van Ettinger, I., Maclntyre, N., Archibald, A.L. \& Ait-Ali, T. (2014)

911 Down-regulation of mechanisms involved in cell transport and maintenance of mucosal

912 integrity in pigs infected with Lawsonia intracellularis. Vet. Res. 45, 55.

913 Sodhi, S.S., Song, K.-D., Ghosh, M., Sharma, N., Lee, S.J., Kim, J.H., Kim, N., Mongre, R.K.,

914 Adhikari, P., Kim, J.Y., Hong, S.P., Oh, S.J. \& Jeong, D.K. (2014) Comparative

915 transcriptomic analysis by RNA-seq to discern differential expression of genes in liver

916 and muscle tissues of adult Berkshire and Jeju Native Pig. Gene 546, 233-242.

917 Spurlock, M.E. \& Gabler, N.K. (2008) The development of porcine models of obesity and the

918 metabolic syndrome. J. Nutr. 138, 397-402.

919 Toedebusch, R.G., Roberts, M.D., Wells, K.D., Company, J.M., Kanosky, K.M., Padilla, J.,

920 Jenkins, N.T., Perfield, J.W., Ibdah, J.A., Booth, F.W. \& Rector, R.S. (2014) Unique

921 transcriptomic signature of omental adipose tissue in Ossabaw swine: a model of

922 childhood obesity. Physiol. Genomics 46, 362-375.

$-40-$ 
923 Trapnell, C., Roberts, A., Goff, L., Pertea, G., Kim, D., Kelley, D.R., Pimentel, H., Salzberg, S.L.,

924 Rinn, J.L. \& Pachter, L. (2012) Differential gene and transcript expression analysis of

925 RNA-seq experiments with TopHat and Cufflinks. Nat Protoc 7, 562-578.

926 Trapnell, C., Williams, B.A., Pertea, G., Mortazavi, A., Kwan, G., van Baren, M.J., Salzberg,

927 S.L., Wold, B.J. \& Pachter, L. (2010) Transcript assembly and quantification by RNA-Seq

928 reveals unannotated transcripts and isoform switching during cell differentiation. Nat.

929 Biotechnol. 28, 511-515.

930 Trayhurn, P. (2005) Endocrine and signalling role of adipose tissue: new perspectives on fat.

$931 \quad$ Acta Physiol. Scand. 184, 285-293.

932 Uenishi, H., Eguchi, T., Suzuki, K., Sawazaki, T., Toki, D., Shinkai, H., Okumura, N., Hamasima,

933 N. \& Awata, T. (2004) PEDE (Pig EST Data Explorer): construction of a database for ESTs

934 derived from porcine full-length cDNA libraries. Nucl. Acids Res. 32, D484-D488.

935 Uenishi, H., Eguchi-Ogawa, T., Shinkai, H., Okumura, N., Suzuki, K., Toki, D., Hamasima, N. \&

936 Awata, T. (2007) PEDE (Pig EST Data Explorer) has been expanded into Pig Expression

937 Data Explorer, including 10147 porcine full-length cDNA sequences. Nucl. Acids Res. 35,

938 D650-D653.

939 Vandesompele, J., De Preter, K., Pattyn, F., Poppe, B., Van Roy, N., De Paepe, A. \& Speleman,

940 F. (2002) Accurate normalization of real-time quantitative RT-PCR data by geometric

941 averaging of multiple internal control genes. Genome Biol. 3, RESEARCHOO34.

942 Varga, O., Harangi, M., Olsson, I. a. S. \& Hansen, A.K. (2010) Contribution of animal models

943 to the understanding of the metabolic syndrome: a systematic overview. Obes Rev 11,

$944 \quad 792-807$.

945 Vicario, M., Skaper, S.D., Negro, A. (2014) The small heat shock protein HspB8: role in

946 nervous system physiology and pathology. CNS Neurol Disord Drug Targets 13, 885-895.

$-41-$ 
947 Wang, R.N., Green, J., Wang, Z., Deng, Y., Qiao, M., Peabody, M., Zhang, Q., Ye, J., Yan, Z.,

948 Denduluri, S., Idowu, O., Li, M., Shen, C., Hu, A., Haydon, R.C., Kang, R., Mok, J., Lee, M.J.,

949 Luu, H.L. \& Shi, L.L. (2014) Bone Morphogenetic Protein (BMP) signaling in development

$950 \quad$ and human diseases. Genes Dis 1, 87-105.

951 Yamada, K., Santo-Yamada, Y. \& Wada, K. (2002) Restraint stress impaired maternal

952 behavior in female mice lacking the neuromedin B receptor (NMB-R) gene. Neurosci.

$953 \quad$ Lett. 330, 163-166.

954 Zhou, C., Zhang, J., Ma, J., Jiang, A., Tang, G., Mai, M., Zhu, L., Bai, L., Li, M. \& Li, X. (2013)

955 Gene expression profiling reveals distinct features of various porcine adipose tissues.

$956 \quad$ Lipids Health Dis 12, 75.

957 Zhou, Z.-Y., Li, A.-M., Adeola, A.C., Liu, Y.-H., Irwin, D.M., Xie, H.-B. \& Zhang, Y.-P. (2014)

958 Genome-Wide Identification of Long Intergenic Noncoding RNA Genes and Their

959 Potential Association with Domestication in Pigs. Genome Biol Evol 6, 1387-1392.

960

$-42-$

Animal Genetics 
961 Supporting information

962 Additional supporting information may be found in the online version of this article.

963 Supplementary Tables are included in the file:

964 TranscriptomeILW_SupplementaryTables.xIsx

965

966 Table S1 - Primers and PCR condition used for the validation.

967 EXT: primer pairs used for the amplification of a larger PCR product

968 INT: primer pairs used for the creation of the standard curve and for the qRT-PCR analysis

969 Table S2 - Number of reads for each sample

970 For each sample is indicated the total raw reads sequenced, total clean reads after the

971 trimming and length filters and total reads mapped to the reference genome. Reported

972 values refer to reads as they were single end (total clean paired reads are half the value in

973 the table). Respective percentages are shown in the last three columns.

974 Table S3 - Types of transcripts expressed in backfat tissue, according to the considered

975 genome annotations.

976 Transcripts, associated to eight Cufflinks class codes (see

977 http://cufflinks.cbcb.umd.edu/manual.html\#class_codes), were classified into three major

978 informative groups.

979 Table S4 - Intergenic transcript annotations. 
980 Table S5 - Transcript coding potential predicted by Coding Potential Calculator (CPC) for

981 intergenic transcripts.

982 Reliable noncoding: CPC score <-1

983 Noncoding: CPC score $-1=>/<=0$

984 Coding: CPC score $>0$

985 Table S6 - Most expressed transcripts (top 75\%) detected in porcine backfat.

986

987 Table S7 - David functional annotation clustering of the most expressed genes.

988 The 10 most relevant clusters are reported

989

$990 \quad$ Table S8 - List of differentially expressed genes detected by Cuffdiff2.

991

992 Table S9 - List of differentially expressed genes detected by DeSeq2.

993

994 Table S10 - List of differentially expressed transcripts detected by Cuffdiff2.

995

996 Table S11 - Transcript coding potential predicted by Coding Potential Calculator (CPC) for

997 the differentially expressed transcripts.

998 Reliable noncoding: CPC score <-1

999 Noncoding: CPC score $-1=>/<=0$

1000 Coding: CPC score $>0$

$$
-44 \text { - }
$$


1001

1002 Figure S1 - Read processing and alignment results.

\section{File: TranscriptomelLW_FigureS1.jpg}

1004 (A) The boxplots show the distribution of the reads considered in different steps and filters

1005 of the computational analysis pipeline, in the 20 considered samples. From left to right we

1006 show the number of raw reads sequenced, of clean reads resulted from the filtering steps,

1007 of reads successfully mapped to the reference genome, and of reads with unique alignment

1008 in the genome. (B) From the left, the bars show the average amounts, in the 20 considered

1009 samples, of reads spliced, aligned to an exon, to an intron, to intergenic regions (according

1010 to the Sus scrofa 10.2 genome annotation), or spanning exon-intron borders. Different

1011 colors indicate the proportion of read aligning to chromosomes (blue), genome scaffolds

1012 (red) or mitochondrial genome (yellow). (C) Number of expressed genes detected in

1013 different chromosomes, in mitochondrial genome (Mt) or in genome scaffolds (S).

1014 Figure S2 - Gene expression distribution in FAT and LEAN groups.

1015 File: TranscriptomelLW_FigureS2.jpg

1016 Cumulative gene expression is shown for the two groups. The figure represents the number

1017 of genes (horizontal axis) required to reach different percentages (vertical axis) of the

1018 overall gene expression. The inner panel focus on the cumulative expression curves for $50 \%$

1019 and $75 \%$ of the expression.

$-45-$ 
1020 Figure S3 - Alignment of the four detected isoforms of PLIN2 gene (red box) with the

1021 porcine and vertebrates transcripts present in Ensembl.

1022 File: TranscriptomelLW_FigureS3.jpg

1023

1024 Figure S4 - Principal component analysis (PCA) based on gene expression profiles.

1025 File: TranscriptomelLW_FigureS4.jpg

1026 The figure presents sample separation according to the two principal components,

1027 explaining most of the gene expression variation in the data. Samples are represented by

1028 dots, with green and orange colours indicating LEAN and FAT samples, respectively in Panel

1029 A) and red and blue indicating females and castrated males in Panel B). The PCA shows a

1030 clear separation of LEAN and FAT samples, with no separation of samples by sex.

1031

- 46 -

Animal Genetics 
1032 Figure captions

1033 Figure 1 - Transcripts and isoforms classification.

\section{TranscriptomelLW_Figure1.jpg}

1035 (A) Expressed transcript were classified, according to current gene annotations, into 8 types,

1036 reported with different colors (see legend) and grouped in three categories: $\mathrm{K}$ (known)

1037 collects transcripts found in reference annotation (yellow); I (isoform) collects alternative

1038 forms of transcripts (red shades); N collects new transcripts from not-annotated loci (green

1039 shades). The pie chart shows the number of transcripts detected, for each type, and their

1040 mutual proportions. Three transcript types of the $\mathrm{N}$ group have few elements (43 intronic; 5

1041 possible polymerase run-on fragments; 3 transcript intron overlap a reference intron on the

1042 opposite strand) and are barely visible in the chart. (B) Transcript length distributions in the

1043 three categories. (C) Transcript expression level distribution for the three categories. (D)

1044 Number of genes (vertical axis) with their number of transcript isoforms detected

1045 (horizontal axis). Genes with only one transcript isoforms detected are the most frequent;

1046 however, genes with up to 31 different isoforms were detected. (E) The proportion of each

1047 transcript type for the transcript isoforms grouped as in (D). Genes with only one isoform

1048 (first bar) are mainly intergenic genes (green part). For genes having more than one isoform

1049 expressed, the proportion of novel isoforms detected increases along with the number of

1050 different isoforms for a gene (red part).

1051

$-47-$ 
1052 Figure 2 - Coding potential of new intergenic transcripts.

\section{TranscriptomelLW_Figure2.jpg}

1054 According to CPC scores, calculated both for the forward and for the reverse complement

1055 sequence, the intergenic transcripts were classified as "coding", "non-coding" and "reliable

1056 non-coding". (A) The pie chart shows numbers and proportions of intergenic transcripts

1057 falling in each category and provides the color code for the figure panels. (B) and (C) show

1058 respectively the distribution of lengths and of expression levels of intergenic transcripts,

1059 binned in the three categories. (D) Percentages of transcripts per category are compared,

1060 considering all the intergenic transcripts and the subset of the intergenic transcripts ranked

1061 within the $10 \%$ most expressed transcripts considering the whole transcriptome.

1062

1063 Figure 3 - Differentially expressed genes and transcripts identified.

\section{TranscriptomelLW_Figure3.jpg}

1065 (A) Intersection of genes resulting differentially expressed (DE) according to DESeq2 and

1066 Cuffdiff2 analysis, and genes with at least one transcript resulting DE according to the

1067 transcript-level Cuffdiff2 analysis. We focused on the transcripts belonging to the 85 genes

1068 commonly identified by all the methods. (B) Proportions of the new and known DETs

1069 resulting higher- and lower-expressed in FAT vs. LEAN samples. (C) Number of DE genes

1070 mapping to chromosomes or to genome scaffolds (S).

$-48-$ 
1071

1072 Figure 4 - qRT-PCR validation of eleven genes differentially expressed according to RNA-

1073 seq data.

\section{TranscriptomelLW_Figure4.jpg}

1075 (A) $\log _{2}$ FC values obtained from RNA-seq, according to Cuffdiff2 estimates, (black bars)

1076 and from qRT-PCR data (grey bars), for the eleven tested genes; (B) scatterplot showing the

1077 good correlation between the $\log _{2}$ FC values calculated with the two experimental

1078 methods.

1079

Figure 5 - Genes differentially expressed between FAT and LEAN animals impact on

1081 specific and connected biological processes.

1082 TranscriptomelLW_Figure5.jpg

1083 Genes differentially expressed in FAT vs. LEAN pigs converge to specific functions that are

1084 more activated or impaired in FAT pigs. Genes and functions upregulated and

1085 downregulated in FAT pigs are shown in red and green shades, respectively. Several genes

1086 more expressed in FAT pigs are linked to fat deposition and lipid metabolism, to adipocyte

1087 differentiation and maturation or to signaling pathways regulating them; FAT pigs show as

1088 well increased expression of genes involved in inflammation and immunity and increased

1089 expression of genes involved in the control of complex behavior, also by inflammation-

1090 mediated secretory activity of adipocytes. Metabolic alterations induce chronic stress in the

1091 adipose tissue. FAT pigs shows under-expression of several genes involved in stress

1092 response by unfolded protein binding and misfolded protein aggregation prevention. The

$$
-49 \text { - }
$$


1093 impairment of these functions might in turn augment inflammation and the consequent

1094 secretory activity and possibly induce senescence.

1095 
1096 Tables

1097

1098 Table 1 - Genetic indexes and phenotypes for BFT and hot carcass weight of the pigs

1099 selected for the transcriptome analysis.

1100

\begin{tabular}{|c|c|c|c|c|c|c|c|c|}
\hline \multirow[b]{2}{*}{ Group } & \multirow{2}{*}{$\begin{array}{c}\text { Sample } \\
\text { ID }\end{array}$} & \multirow[b]{2}{*}{ Sex } & \multirow{2}{*}{$\begin{array}{c}\text { Day of } \\
\text { slaughter }\end{array}$} & \multirow{2}{*}{$\begin{array}{c}\text { Slaughter } \\
\text { weight }(\mathrm{kg}) \\
(*)\end{array}$} & \multirow{2}{*}{$\begin{array}{c}\text { BFT } \\
\text { phenotype } \\
(\mathrm{mm})\end{array}$} & \multicolumn{3}{|c|}{ BFT EBV } \\
\hline & & & & & & & Mean & SD \\
\hline \multirow{10}{*}{ FAT } & 477 & $M$ & 6 & 120 & 43 & 7.36 & \multirow{10}{*}{5.22} & \multirow{10}{*}{1.3} \\
\hline & 476 & $\mathrm{~F}$ & 6 & 119 & 37 & 7.17 & & \\
\hline & 474 & $M$ & 2 & 113 & 38 & 6.03 & & \\
\hline & 482 & $\mathrm{~F}$ & 9 & & 42 & 5.75 & & \\
\hline & 478 & $\mathrm{~F}$ & 7 & 118 & 33 & 5.05 & & \\
\hline & 516 & $\mathrm{~F}$ & 3 & 115 & 36 & 4.88 & & \\
\hline & 479 & $M$ & 8 & & 41 & 4.76 & & \\
\hline & 483 & $\mathrm{~F}$ & 10 & 119 & 38 & 4.41 & & \\
\hline & 489 & $M$ & 18 & 108 & 35 & 3.54 & & \\
\hline & 484 & $M$ & 15 & 128 & 35 & 3.27 & & \\
\hline \multirow{10}{*}{ LEAN } & 490 & $M$ & 19 & 113 & 24 & -6.46 & \multirow{10}{*}{-8.63} & \multirow{10}{*}{1.4} \\
\hline & 473 & $\mathrm{~F}$ & 2 & 132 & 23 & -7.54 & & \\
\hline & 487 & $M$ & 18 & 110 & 23 & -7.61 & & \\
\hline & 517 & $M$ & 4 & 117 & 20 & -7.71 & & \\
\hline & 485 & $\mathrm{~F}$ & 17 & 126 & 20 & -7.82 & & \\
\hline & 475 & $M$ & 5 & 119 & 20 & -8.03 & & \\
\hline & 481 & $\mathrm{M}$ & 9 & & 22 & -9.91 & & \\
\hline & 486 & $\mathrm{~F}$ & 17 & 123 & 19 & -10.27 & & \\
\hline & 488 & $\mathrm{~F}$ & 18 & 128 & 19 & -10.37 & & \\
\hline & 480 & $\mathrm{~F}$ & 9 & & 16 & \begin{tabular}{|l|}
-10.59 \\
\end{tabular} & & \\
\hline
\end{tabular}

1101 EBV: estimated breeding value

1102 BFT: backfat thickness.

$1103(*)$ slaughter weight: the hot carcass slaughter weight is reported. For four animals the

1104 weight is not available due to a problem of the automatic recording system at the

1105 slaughterhouse.

1106

$-51-$

Animal Genetics 
Table 2 - List of the DE genes and transcripts.

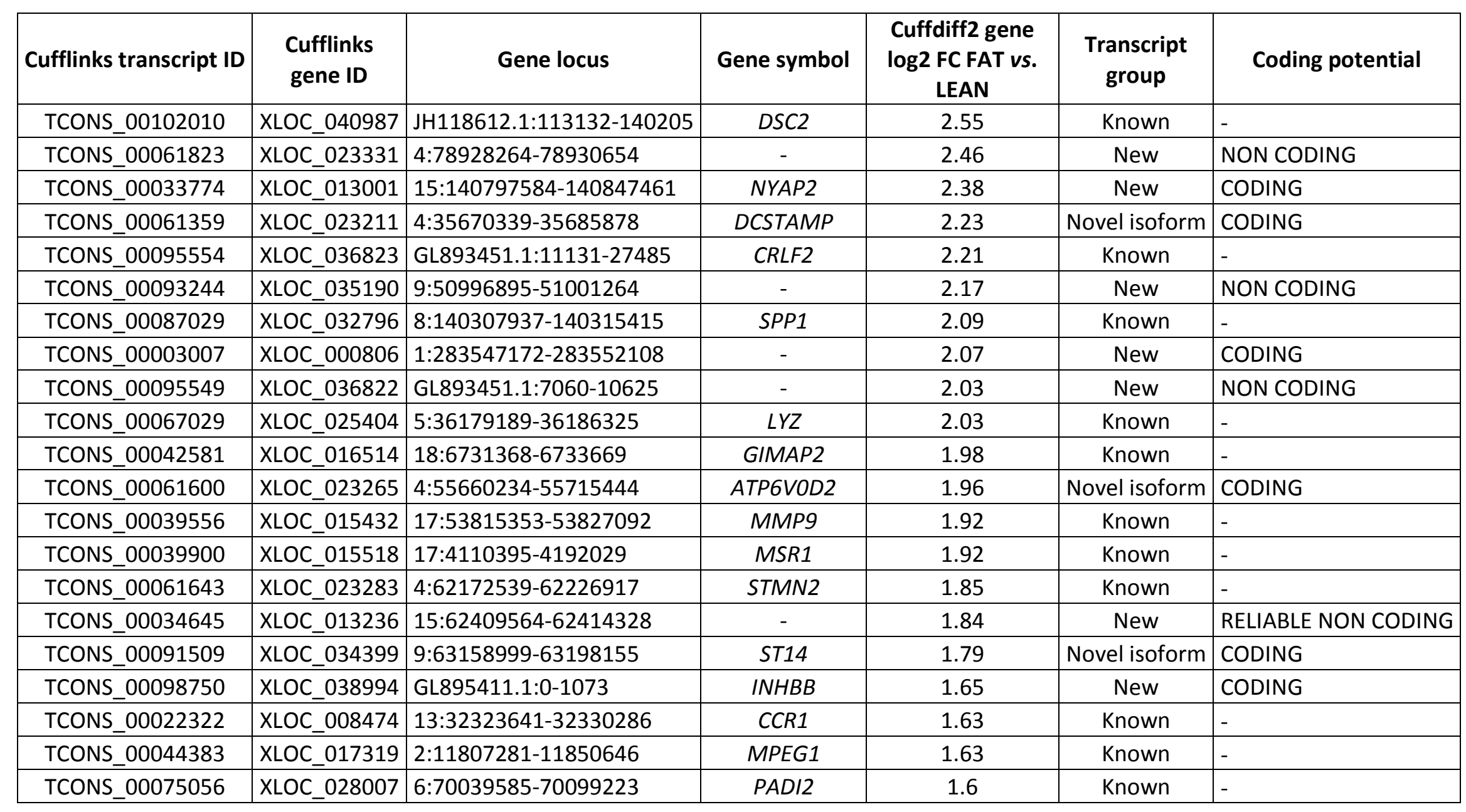

$-52-$ 


\begin{tabular}{|c|c|c|c|c|c|c|}
\hline TCONS_00095875 & XLOC_037025 & | GL893645.1:0-307 & - & 1.57 & New & RELIABLE NON CODING \\
\hline TCONS_00084869 & XLOC_032187 & 8:71288921-71302169 & $A M B N$ & 1.56 & Known & - \\
\hline TCONS_00033691 & XLOC_012975 & $15: 133452328-133456736$ & $S L C 11 A 1$ & 1.56 & Known & - \\
\hline TCONS_00089513 & XLOC_033895 & 9:90266412-90348498 & $S C I N$ & 1.55 & Known & - \\
\hline TCONS_00042660 & XLOC_016535 & 18:8306789-8313120 & - & 1.52 & New & CODING \\
\hline TCONS_00059834 & XLOC_022860 & 4:99905518-99915176 & CD1A & 1.52 & Novel isoform & CODING \\
\hline TCONS_00059837 & XLOC_022860 & 4:99905518-99915176 & $C D 1 A$ & 1.52 & Known & - \\
\hline TCONS_00093519 & XLOC_035465 & 9:101443296-101443885 & GPNMB & 1.46 & New & NON CODING \\
\hline TCONS_00098157 & XLOC_038614 & GL894967.1:126-517 & GPNMB & 1.42 & New & CODING \\
\hline TCONS_00018804 & XLOC_007247 & 12:23439824-23441829 & - & 1.4 & New & CODING \\
\hline TCONS_00103084 & XLOC_041497 & $\mathrm{X}: 37303173-37393818$ & CYBB & 1.38 & Known & - \\
\hline TCONS_00065337 & XLOC_024931 & 5:52504178-52625145 & BCAT1 & 1.37 & Novel isoform & CODING \\
\hline TCONS_00098113 & XLOC_038589 & GL894923.1:47-563 & GPNMB & 1.36 & New & CODING \\
\hline TCONS_00002441 & XLOC_000664 & 1:227333991-227356844 & PLIN2 & 1.32 & Novel isoform & CODING \\
\hline TCONS_00044392 & XLOC_017322 & 2:12191483-12243400 & $\angle P X N$ & 1.31 & Known & - \\
\hline TCONS_00084565 & XLOC_032101 & 8:33970571-33982450 & UCHL1 & 1.27 & Novel isoform & CODING \\
\hline TCONS_00067389 & XLOC_025495 & 5:64579162-64590512 & OLR1 & 1.26 & Known & - \\
\hline TCONS_00059747 & XLOC_022835 & 4:97720982-97736619 & CD48 & 1.25 & Known & - \\
\hline TCONS_00028769 & XLOC_011055 & 14:143745489-143752509 & GMFG & 1.23 & Known & - \\
\hline TCONS_00029056 & XLOC_011139 & 14:8804077-8816800 & STC1 & 1.23 & Novel isoform & CODING \\
\hline TCONS_00098643 & XLOC_038938 & GL895339.1:13269-61205 & COTL1 & 1.15 & Known & - \\
\hline TCONS_00100592 & XLOC_040068 & GL896326.1:1999-3913 & ACP5 & 1.13 & Known & - \\
\hline TCONS_00096837 & XLOC_037668 & GL894123.1:0-400 & CD163 & 1.13 & New & CODING \\
\hline TCONS_00097297 & XLOC_037990 & GL894401.1:0-471 & CD163 & 1.13 & New & CODING \\
\hline TCONS_00005002 & XLOC_001331 & 1:125897935-125953413 & $A Q P 9$ & 1.09 & Known & - \\
\hline TCONS_00096863 & XLOC_037686 & GL894145.1:0-401 & CD163 & 1.09 & New & CODING \\
\hline TCONS_00071337 & XLOC_027094 & 6:74616232-74621248 & $C 1 Q C$ & 1.08 & Known & - \\
\hline
\end{tabular}




\begin{tabular}{|c|c|c|c|c|c|c|}
\hline TCONS_00012469 & XLOC_005058 & | 11:21534980-21685851 & $\angle C P 1$ & 1.07 & Novel isoform & CODING \\
\hline TCONS_00079920 & XLOC_030238 & 7:94900207-94906867 & $\begin{array}{c}\text { AKAP5 } \\
\text { LOC100153460 }\end{array}$ & 1.06 & Novel isoform & CODING \\
\hline TCONS_00041537 & XLOC_016257 & 18:6613761-6621027 & GIMAP4 & 1.06 & Known & - \\
\hline TCONS_00030401 & XLOC_011444 & 14:71516962-71521335 & $E G R 2$ & 1.05 & Known & - \\
\hline TCONS_00030878 & XLOC_011579 & 14:117265093-117349965 & $B L N K$ & 1.04 & Known & - \\
\hline TCONS_00081915 & XLOC_030757 & 7:54395230-54406136 & $B C L 2 A 1$ & 1.01 & Known & - \\
\hline TCONS_00041554 & XLOC_016261 & 18:6872940-6875292 & GIMAP1 & 1 & Known & - \\
\hline TCONS_00085005 & XLOC_032236 & 8:79743274-79751980 & SFRP2 & 0.99 & Known & - \\
\hline TCONS_00098919 & XLOC_039115 & GL895590.1:0-1327 & GPNMB & 0.91 & New & NON CODING \\
\hline TCONS_00068526 & XLOC_026077 & 5:52625315-52630242 & BCAT1 & 0.89 & New & RELIABLE NON CODING \\
\hline TCONS_00062959 & XLOC_023614 & 4:119674090-119703427 & CD53 & 0.78 & Known & - \\
\hline TCONS_00081898 & XLOC_030753 & 7:53623061-53644262 & CTSH & 0.78 & Known & - \\
\hline TCONS_00060570 & XLOC_023035 & 4:119013307-119039899 & ADORA3 & 0.74 & Known & - \\
\hline TCONS_00052401 & XLOC_020144 & 3:11035819-11055510 & LAT2 & 0.71 & Known & - \\
\hline TCONS_00004118 & XLOC_001095 & 1:35133812-35137388 & CTGF & 0.68 & Known & - \\
\hline TCONS_00045043 & XLOC_017499 & 2:59214054-59218018 & IFI30 & 0.65 & Known & - \\
\hline TCONS_00004124 & XLOC_001096 & 1:35240242-35281384 & ENPP1 & 0.62 & Known & - \\
\hline TCONS_00062884 & XLOC_023592 & 4:116704501-116707235 & OLFML3 & -0.54 & Known & - \\
\hline TCONS_00035484 & XLOC_013426 & $15: 131680309-131684630$ & IGFBP5 & -0.65 & Known & - \\
\hline
\end{tabular}




\begin{tabular}{|c|l|l|c|c|c|c|} 
TCONS_00101718 & XLOC_040809 & JH118426.1:306724-312138 & - & -0.77 & New & RELIABLE NON CODING \\
\hline TCONS_00063805 & XLOC_024145 & $4: 77261119-77264781$ & - & -0.77 & New & NON CODING \\
\hline TCONS_00050164 & XLOC_018733 & $2: 124815021-124828122$ & CDO1 & -0.9 & Novel isoform & CODING \\
\hline TCONS_00101559 & XLOC_040715 & GL896532.1:212-2567 & ADSSL1 & -1.02 & New & NON CODING \\
\hline TCONS_00079927 & XLOC_030240 & $7: 94987617-94990126$ & HSPA2 & -1.1 & Known & - \\
\hline TCONS_00083805 & XLOC_031620 & $7: 66542203-66555641$ & - & -1.18 & New & CODING \\
\hline TCONS_00041725 & XLOC_016313 & $18: 15292592-15295178$ & - & -1.61 & New & CODING \\
\hline TCONS_00048853 & XLOC_018425 & $2: 65175406-65180520$ & DNAJB1 & -1.66 & Novel isoform & CODING \\
\hline TCONS_00029533 & XLOC_011248 & $14: 35688332-35701411$ & HSPB8 & -1.81 & Known & - \\
\hline TCONS_00094194 & XLOC_036009 & GL892492.1:0-3540 & HSPA1B & -2.32 & New & NON CODING \\
\hline TCONS_00101505 & XLOC_040677 & GL896522.1:9039-10877 & HSPA1A & -2.57 & New & RELIABLE NON CODING \\
\hline TCONS_00098059 & XLOC_038555 & GL894890.1:5-696 & HSP70 & -3.44 & New & NON CODING \\
\hline
\end{tabular}


Table 3 - David functional annotation clustering obtained considering the significant Biological Processes GO terms (Benjamini adjusted Pvalues $<0.05$ ) of genes more expressed in FAT than in LEAN animals.

\begin{tabular}{|c|c|c|}
\hline Annotation Cluster 1 & & Enrichment Score: 7.0 \\
\hline Term & Count & Genes \\
\hline GO:0006954 inflammatory response & 12 & C1QA, SLC11A1, CYBB, ADORA3, OLR1, HMOX1, CCR1, LYZ, C1QC, BLNK, CD163, SPP1 \\
\hline GO:0006952 defense response & 15 & $\begin{array}{l}\text { ADORA3, OLR1, CCR1, LYZ, COTL1, C1QC, CD163, INHBB, CD48, C1QA, SLC11A1, CYBB, HMOX1, } \\
\text { SPP1, BLNK }\end{array}$ \\
\hline GO:0009611 response to wounding & 14 & $\begin{array}{l}\text { ADORA3, PLEK, OLR1, CCR1, LYZ, C1QC, CD163, C1QA, SLC11A1, CYBB, CTGF, HMOX1, SPP1, } \\
\text { BLNK }\end{array}$ \\
\hline $\begin{array}{l}\text { GO:0009605 response to external } \\
\text { stimulus }\end{array}$ & 17 & $\begin{array}{l}\text { ADORA3, PLEK, OLR1, CCR1, LYZ, C1QC, CD163, INHBB, C1QA, SLC11A1, CYBB, CTGF, SFRP2, } \\
\text { HMOX1, STC1, SPP1, BLNK }\end{array}$ \\
\hline GO:0050896 response to stimulus & 29 & $\begin{array}{l}\text { ADORA3, AQP9, ENPP1, CCR1, UCHL1, ACP5, C1QC, CD48, SLC11A1, PLIN2, CTGF, HMOX1, } \\
\text { FCER1G, BLNK, SPP1, EGR2, OLR1, PLEK, LYZ, CD1A, COTL1, CD163, INHBB, C1QA, CYBB, LAT2, } \\
\text { SFRP2, STC1, LCP1 }\end{array}$ \\
\hline GO:0006950 response to stress & 19 & $\begin{array}{l}\text { ADORA3, AQP9, PLEK, OLR1, CCR1, UCHL1, LYZ, COTL1, C1QC, CD163, INHBB, CD48, C1QA, } \\
\text { SLC11A1, CYBB, CTGF, HMOX1, SPP1, BLNK }\end{array}$ \\
\hline Annotation Cluster 2 & & Enrichment Score: 2.7 \\
\hline Term & Count & Genes \\
\hline GO:0001775 cell activation & 7 & CD48, SLC11A1, LAT2, PLEK, LCP1, BLNK, GIMAP1 \\
\hline $\begin{array}{l}\text { GO:0002274 myeloid leukocyte } \\
\text { activation }\end{array}$ & 4 & CD48, SLC11A1, LAT2, GIMAP1 \\
\hline GO:0046649 /ymphocyte activation & 6 & CD48, SLC11A1, LAT2, LCP1, BLNK, GIMAP1 \\
\hline Annotation Cluster 3 & & Enrichment Score: 2.4 \\
\hline
\end{tabular}




\begin{tabular}{|c|c|c|}
\hline Term & Count & Genes \\
\hline $\begin{array}{l}\text { GO:0048583 regulation of response } \\
\text { to stimulus }\end{array}$ & 10 & C1QA, SLC11A1, LAT2, PLEK, ENPP1, HMOX1, FCER1G, C1QC, SPP1, GIMAP1 \\
\hline $\begin{array}{l}\text { GO:0050776 regulation of immune } \\
\text { response }\end{array}$ & 7 & C1QA, SLC11A1, LAT2, HMOX1, FCER1G, C1QC, GIMAP1 \\
\hline $\begin{array}{l}\text { GO:0050778 positive regulation of } \\
\text { immune response }\end{array}$ & 6 & C1QA, SLC11A1, LAT2, FCER1G, C1QC, GIMAP1 \\
\hline $\begin{array}{l}\text { GO:0002443 leukocyte mediated } \\
\text { immunity }\end{array}$ & 5 & C1QA, SLC11A1, LAT2, FCER1G, C1QC \\
\hline $\begin{array}{l}\text { GO:0002682 regulation of immune } \\
\text { system process }\end{array}$ & 8 & C1QA, SLC11A1, LAT2, HMOX1, SCIN, FCER1G, C1QC, GIMAP1 \\
\hline Annotation Cluster 4 & & Enrichment Score: 2.0 \\
\hline Term & Count & Genes \\
\hline GO:0060348 bone development & 6 & $A M B N, C T G F, A C P 5, S T C 1, G P N M B, S P P 1$ \\
\hline GO:0031214 biomineral formation & 4 & $A M B N$, ENPP1, GPNMB, SPP1 \\
\hline GO:0001503 ossification & 5 & $A M B N, C T G F, S T C 1, G P N M B, S P P 1$ \\
\hline $\begin{array}{l}\text { GO:0001501 skeletal system } \\
\text { development }\end{array}$ & 7 & $A M B N, C T G F, M M P 9, A C P 5, S T C 1, G P N M B, S P P 1$ \\
\hline Annotation Cluster 5 & & Enrichment Score: 1.6 \\
\hline Term & Count & Genes \\
\hline GO:0001775 cell activation & 7 & CD48, SLC11A1, LAT2, PLEK, LCP1, BLNK, GIMAP1 \\
\hline
\end{tabular}



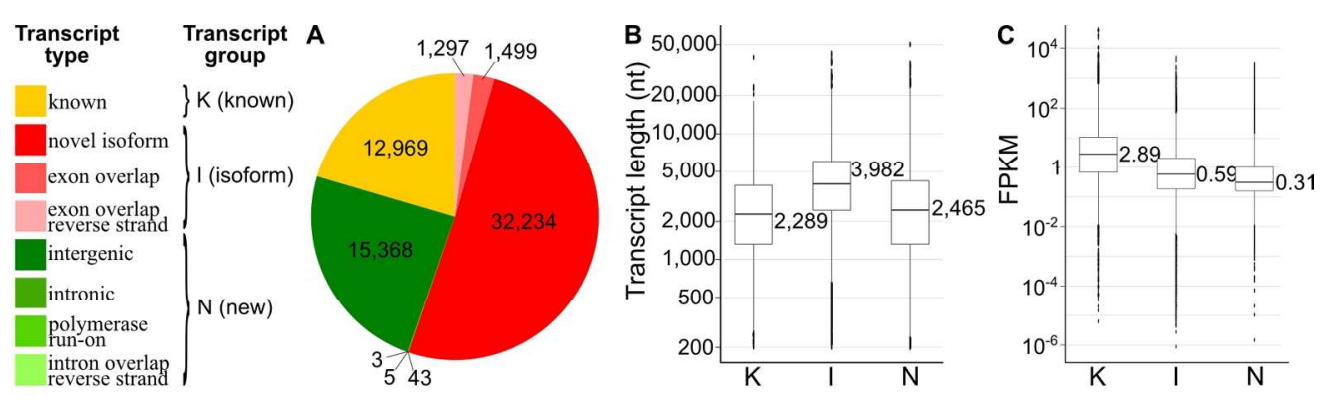

\section{D $12,500-12,138$}
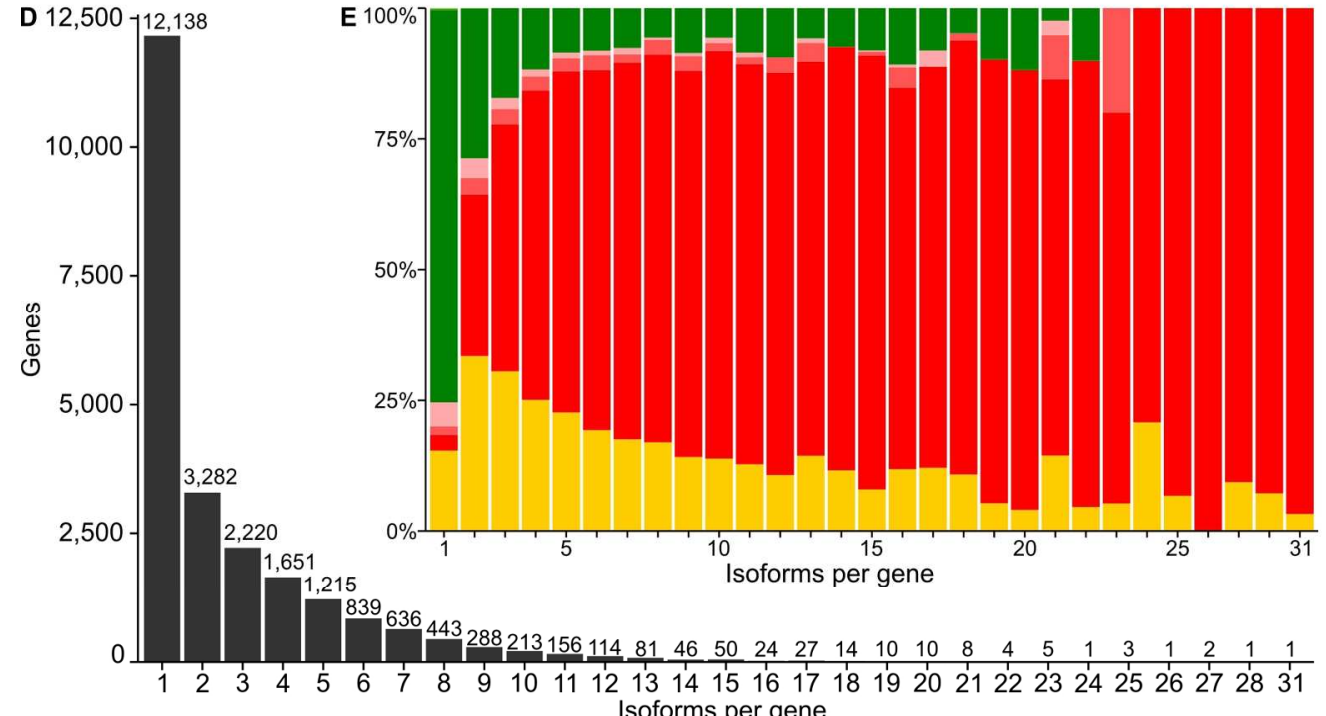

Figure 1 
A CPC classification

B Transcript length (nt)

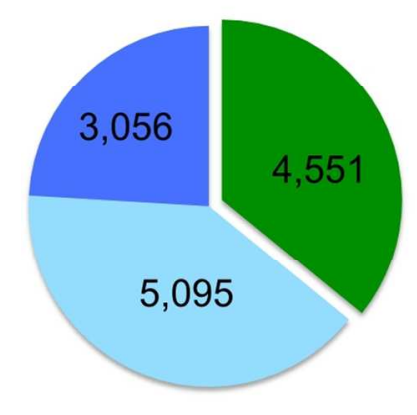

$\square$ Reliable non-coding

$\square$ Non-coding

$\square$ Coding
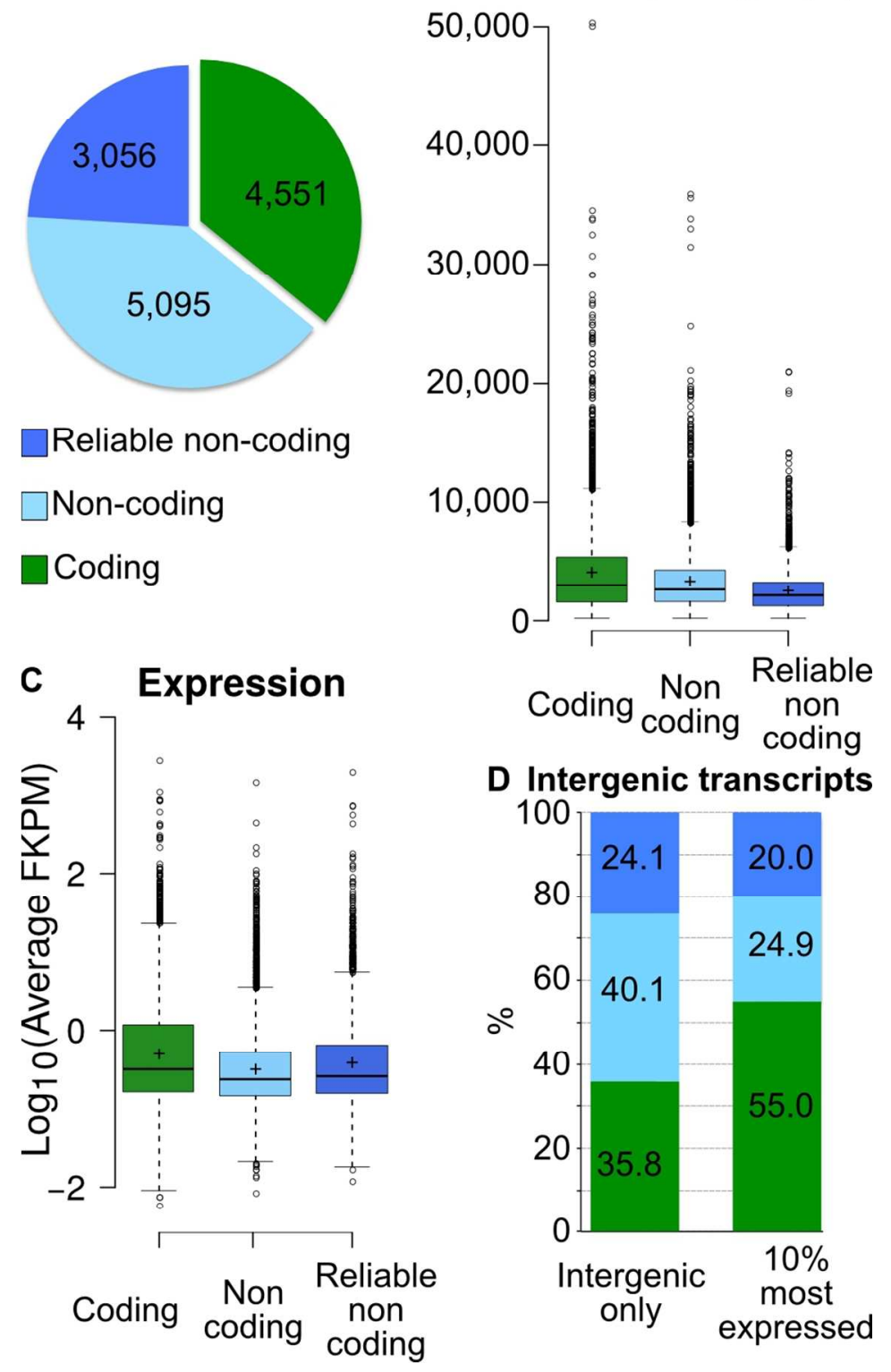

Figure 2 


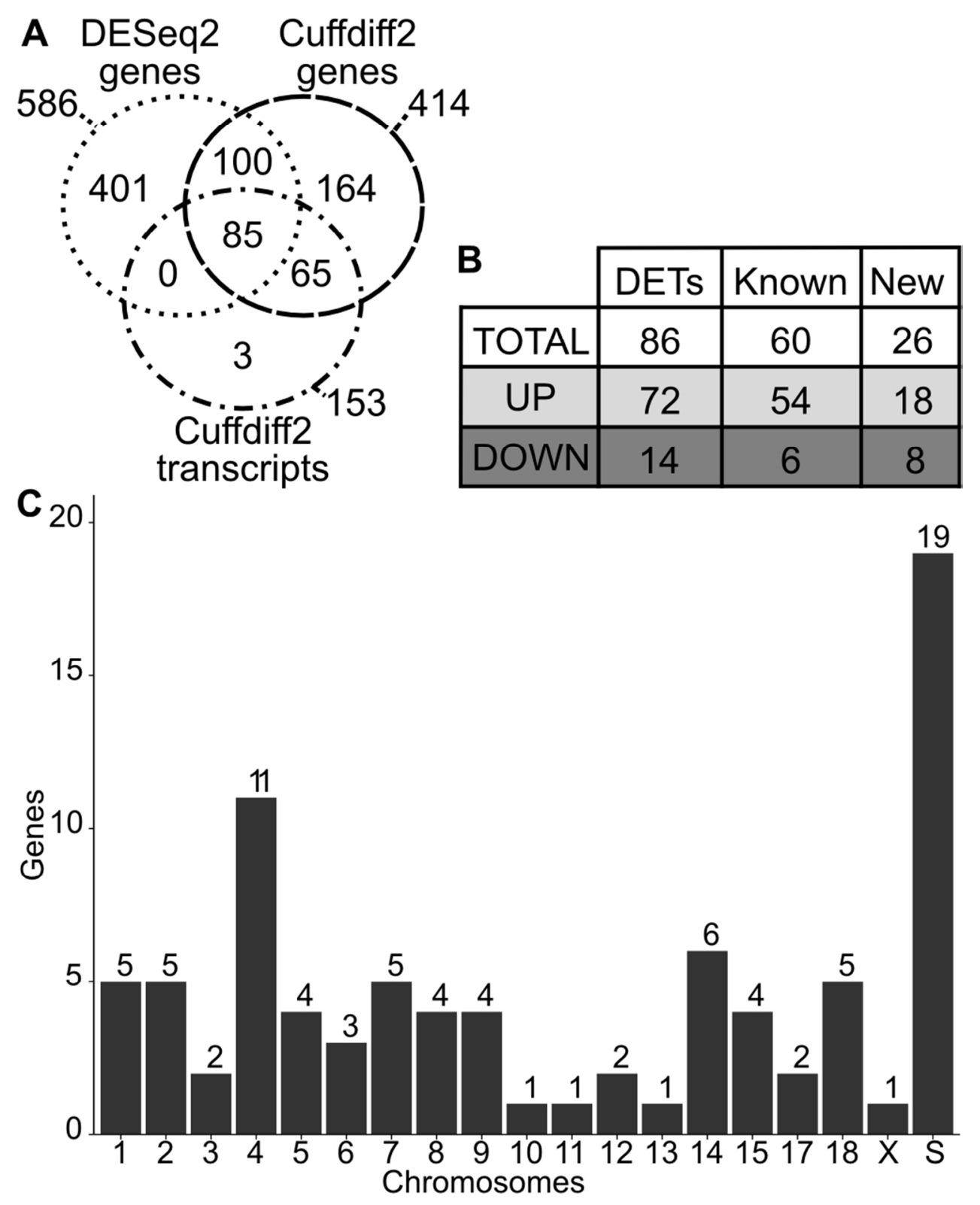

Figure 3

$85 \times 105 \mathrm{~mm}(300 \times 300$ DPI $)$ 

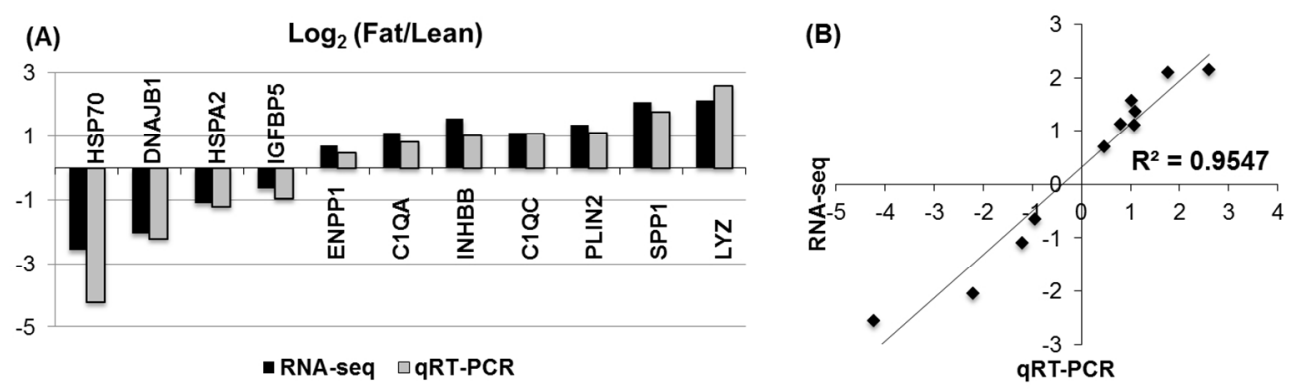

Figure 4

$242 \times 73 \mathrm{~mm}(150 \times 150 \mathrm{DPI})$ 


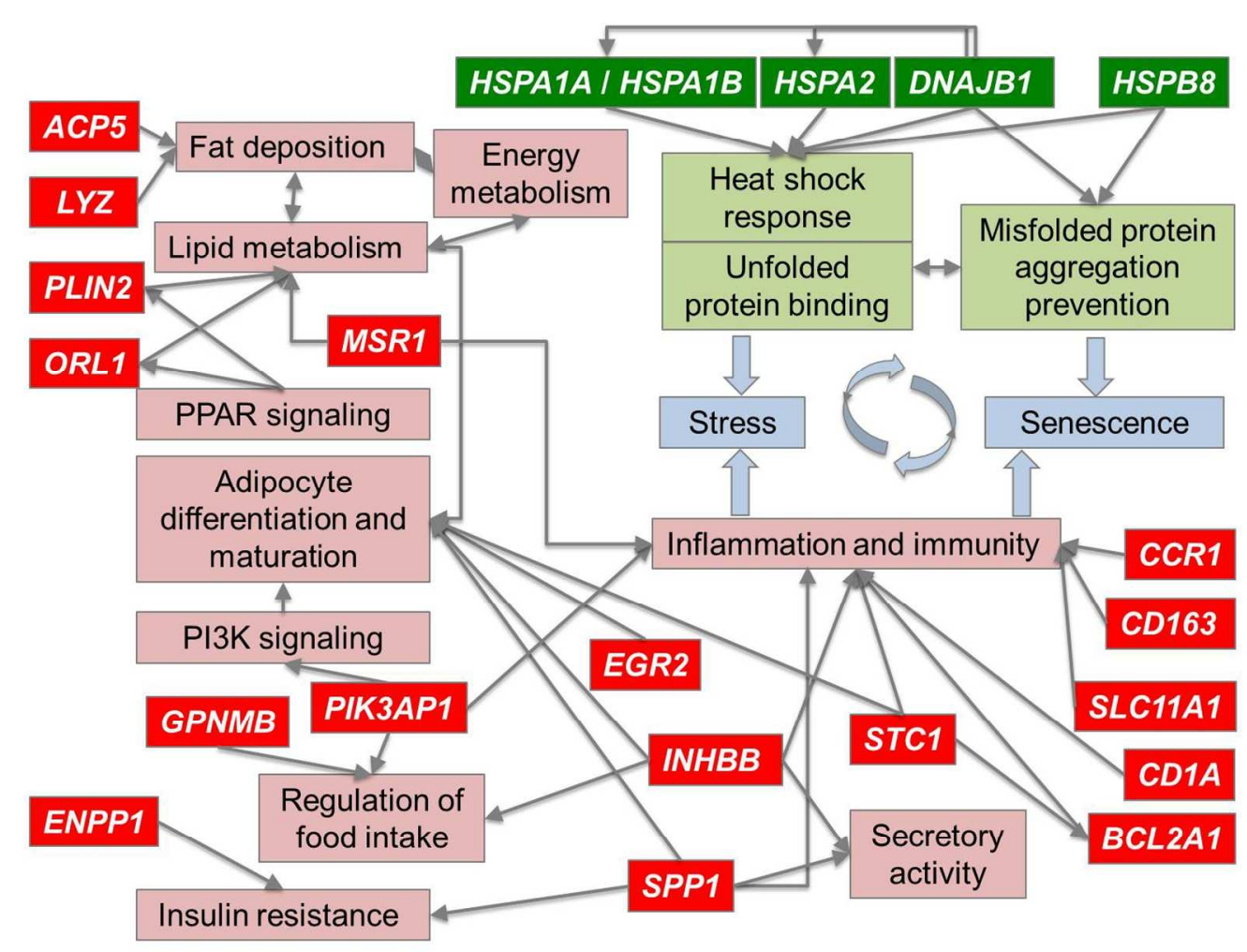

Figure 5

$249 \times 190 \mathrm{~mm}(150 \times 150 \mathrm{DPI})$ 